NASA

Technical

Paper

2385

January 1985

\title{
A Comparative Study of the Nonuniqueness Problem of the Potential Equation
}

M. D. Salas,

A. Jameson,

and R. E. Melnik

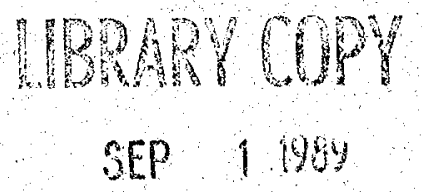

LANGLEY RESEARCH CENTER ISAARY NASA, BAMPOOM, VR 
31176013317756 
NASA

Technical

Paper

2385

1985

\section{A Comparative Study of the Nonuniqueness Problem of the Potential Equation}

M. D. Salas

Langley Research Center

Hampton, Virginia

A. Jameson

Princeton University

Princeton, New Jersey

R. E. Melnik

Grumman Aerospace Corporation

Bethpage, New York 



\section{Summary}

The nonuniqueness problem occurring at transonic speeds with the conservative potential equation is investigated numerically. Evidence is given supporting the thesis that the nonuniqueness problem is inherent in the potential differential equation rather than a consequence of the finite-difference approximation. Results are presented from an extensive comparative study between potential and Euler calculations for flow past two-dimensional airfoil profiles. This study indicates that the nonuniqueness problem is not an inviscid phenomenon, but results from the approximate treatment of shock waves inherent in the conservative potential model. A more restrictive bound on the limit of validity of the conservative potential model is suggested.

\section{Introduction}

When the tangential stresses acting on a volume of fluid are small compared with the normal pressures exerted by the fluid, the fluid may be assumed to be inviscid. The mathematical expressions describing the conservation of mass, momentum, and energy of an inviscid flow are known as the Euler equations. In the absence of rotational effects, the inviscid flow can be described by a single conservation law, the conservation of mass, which can be expressed in terms of a function $\Phi$, the velocity potential. The irrotational assumption is not always valid. Rotational effects can occur in an inviscid flow as a result of variations in free-stream stagnation conditions, because of the presence of shock waves, or because of satisfying boundary conditions, as for example in the case of a lifting delta wing with a sharp leading edge. For flow past two-dimensional airfoils immersed in a uniform upstream far field, rotational effects are introduced at shock waves. Strictly speaking, within the inviscid assumption, these flows are governed by the Euler equations. However, if shock waves are sufficiently weak, the irrotational assumption should provide a reasonable approximation of the flow field. Shock waves, under this approximation, are replaced by sharp isentropic recompressions which approximate the true Rankine-Hugoniot shock up to second order in terms of the shock wave strength.

Under the irrotational assumption, the conservation of mass equation can be written in quasi-linear or nonconservative form as

$$
\left.\begin{array}{l}
\left(a^{2}-\Phi_{x}^{2}\right) \Phi_{x x}-2 \Phi_{x} \Phi_{y} \Phi_{x y}+\left(a^{2}-\Phi_{y}^{2}\right) \Phi_{y y}=0 \\
a=a\left(\Phi_{x}^{2}+\Phi_{y}^{2}\right)
\end{array}\right\}
$$

or in conservation form as

$$
\left.\begin{array}{c}
\left(\rho \Phi_{x}\right)_{x}+\left(\rho \Phi_{y}\right)_{y}=0 \\
\rho=\rho\left(\Phi_{x}^{2}+\Phi_{y}^{2}\right)
\end{array}\right\}
$$

where $a$ is the speed of sound, $\rho$ is the density, $\Phi$ is the potential, and the subscripts denote partial derivatives. In the absence of shock waves, both forms of the equation are equivalent. When shock waves are present, use of the conservative form in conjunction with an "entropy" condition guarantees (see ref. 1) that the proper ${ }^{1}$ shock jump condition is satisfied. On the other hand, use of the nonconservative form leads to an ambiguous jump across the shock waves. With few exceptions, present-day state-of-the-art working transonic potential equation codes use the conservative form.

For subcritical flows, Bers (ref. 2) has shown that the solution for flow past an airfoil section satisfying the Kutta condition is unique. In the transonic regime, little has been proven rigorously in terms of uniqueness. However, in a recent paper (ref. 3), Steinhoff and Jameson showed that numerical solutions of the full potential equation in conservative form for flow past an airfoil are not unique. In the same paper, a meticulous study was conducted which indicated that the anomaly is inherent in the partial differential equation and not a result of its discrete representation. Having reached this conclusion, the authors went on to conjecture the possibility of the anomaly being (1) an inviscid phenomenon, perhaps inherent to the more exact Euler equations; and (2) perhaps a contributing factor in the occurrence of buffeting.

Given the preeminent position today of the potential approximation in the transonic range for the design and analysis of airfoil profiles, as documented in reference 4 , it is unnecessary to dwell on the importance of investigating the conjectures made in reference 3 . Such an investigation is the purpose of this paper. The paper is divided into three main parts. First, a brief review of Steinhoff and Jameson's study and some new findings made in the course of this work are presented. Second, the Euler code described in reference 5 is introduced, and both improvements and the validation procedure used to determine its accuracy are described. Finally, results from a comparative study of the nonuniqueness problem for the potential and Euler models are presented and discussed.

This paper provides results which are more comprehensive than the preliminary results reported in reference 6 and corrects some errors in an earlier version by the same title, reference 7 .

\section{Symbols}

$\begin{array}{ll}a & \text { local speed of sound } \\ C_{p} & \text { pressure coefficient }\end{array}$

1 By proper, we mean consistent with the mathematical model being solved. In this case, the model requires the conservation of mass. 


\begin{tabular}{|c|c|}
\hline$c$ & airfoil chord \\
\hline$c_{d}$ & section drag coefficient \\
\hline$c_{l}$ & section lift coefficient \\
\hline$c_{m}$ & section pitching-moment coefficient \\
\hline$D$ & $\begin{array}{l}\text { damping operator defined by equa- } \\
\text { tion }(6)\end{array}$ \\
\hline$D_{X}$ & $\begin{array}{l}\text { damping operator defined by equa- } \\
\text { tion }(7)\end{array}$ \\
\hline$D_{Y}$ & $\begin{array}{l}\text { damping operator defined by equa- } \\
\text { tion }(8)\end{array}$ \\
\hline$d$ & term defined by equation (9) \\
\hline$h$ & cell area \\
\hline$J_{\max }$ & number of cells in $Y$-direction \\
\hline$L$ & wind-tunnel half-height \\
\hline$M$ & Mach number \\
\hline$r$ & $\begin{array}{l}\text { radial distance measured from center of } \\
\text { airfoil }\end{array}$ \\
\hline$S$ & similarity entropy \\
\hline$t$ & time \\
\hline$u$ & component of velocity in $x$-direction \\
\hline$v$ & component of velocity in $y$-direction \\
\hline$w$ & vector of conservative unknowns \\
\hline$X$ & $\begin{array}{l}\text { computational coordinate along ring of } \\
\text { "O" mesh }\end{array}$ \\
\hline$x$ & Cartesian coordinate \\
\hline$Y$ & $\begin{array}{l}\text { computational coordinate normal to ring } \\
\text { of "O" mesh }\end{array}$ \\
\hline$y$ & Cartesian coordinate \\
\hline$\alpha$ & angle of attack \\
\hline$\beta$ & compressibility factor, $\left(1-M_{\infty}^{2}\right)^{1 / 2}$ \\
\hline$\Gamma$ & circulation \\
\hline$\gamma$ & ratio of specific heats \\
\hline$\Delta$ & increment \\
\hline$\varepsilon^{(2)}$ & $\begin{array}{l}\text { second-order artificial damping } \\
\text { coefficient }\end{array}$ \\
\hline$\varepsilon^{(4)}$ & $\begin{array}{l}\text { fourth-order artificial damping } \\
\text { coefficient }\end{array}$ \\
\hline$\theta$ & $\begin{array}{l}\text { polar angle measured counterclockwise } \\
\text { from trailing edge }\end{array}$ \\
\hline$\kappa$ & term defined by equation (13) \\
\hline
\end{tabular}

$\begin{array}{ll}\rho & \text { density } \\ \tau & \text { ratio of airfoil thickness to airfoil chord } \\ \Phi & \text { velocity potential } \\ \phi & \text { reduced velocity potential } \\ & \phi=\Phi-\left(a_{\infty} M_{\infty}\right)(x \cos \alpha+y \sin \alpha) \\ \chi & \text { transonic similarity, } \\ & \left(1-M_{\infty}^{2}\right) /\left[\tau(\gamma+1) M_{\infty}^{2}\right]^{2 / 3}\end{array}$

Subscripts:

$\begin{array}{ll}d & \text { value downstream from shock wave } \\ F & \text { far-field value } \\ i & \text { cell index in } X \text {-direction } \\ j & \text { cell index in } Y \text {-direction } \\ o & \text { value at which nonuniqueness occurs } \\ s & \text { value upstream from shock wave } \\ \infty & \text { free-stream value }\end{array}$

\section{Review of the Potential Anomaly}

While introducing lifting capability to the fullpotential, conservative, nonlifting, multigrid code described by Jameson in reference 8 , Steinhoff and Jameson (ref. 3) discovered that three converged solutions could be obtained for a symmetric airfoil at zero angle of attack and a fixed free-stream Mach number. These solutions consisted of a zero-lift symmetric solution and two mirror-image asymmetric solutions with large absolute levels of lift. (See figs. 1 and 2 of ref. 3.) The latter two solutions could be obtained by perturbing the zero-lift solution, which was unstable. The multigrid capability of the code made it feasible to converge all three solutions to machine accuracy. ${ }^{2}$ This unquestionably demonstrated the multiplicity of solutions for the discrete system of equations. To determine if the anomaly was characteristic of the partial differential equation being modeled, the following tests were made in reference 3 with FLO 36 :

1. The solutions were checked to determine if they had the expected far-field decay corresponding to a vortex and a doublet in a uniform stream (ref. 9). They did. In addition, the effect of the location of the outer boundary was investigated. It affected the lift level slightly, but the nonuniqueness problem persisted.

2. Originally, the anomaly was observed for an airfoil with nonzero trailing-edge included angle. To determine the effect of imposing the Kutta condition, a Joukowski profile was investigated. The

\footnotetext{
2 Residuals were of the order of $10^{-12}$ in a CDC ${ }^{\circledR}$ CYBER 203 computer for the cases reported here.
} 
Joukowski profile, with its cusped trailing edge, requires a smooth matching of the pressures on the upper and lower surfaces. The numerical solutions showed the proper trailing-edge behavior, but again the anomaly persisted.

3. The convergence of these solutions with mesh refinement was investigated by using grids with $96 \times 24$, $128 \times 32,192 \times 48,256 \times 64$, and $384 \times 192$ cells in the $X$ - and $Y$-directions, respectively. All solutions were found to change little with mesh refinement.

4. The effect of artificial viscosity was tested by using both first- and second-order accurate expressions for this term, with no effect on the anomaly.

5. Since all calculations were originally made with an "O" mesh defined by a conformal circle mapping, calculations were tried with a " $\mathrm{C}$ " mesh. The multiple solutions were also observed with the " $\mathrm{C}$ " mesh.

6. Similar results were obtained with the conservative finite-difference, potential code described in reference 10.

In addition, during the course of the present investigation, we found the following results:

1. The multiple solutions also occurred with the conservative potential code described in reference 11 (TAIR).

2. A standard feature of these potential codes is the requirement that the reduced far-field potential $\phi_{F}$ satisfy the leading term of the compressible vortex solution; namely,

$$
\begin{aligned}
\phi_{F} & =\frac{\Gamma}{2 \pi} \tan ^{-1}[\beta \tan (\theta-\alpha)] \\
\beta & =\left(1-M_{\infty}^{2}\right)^{1 / 2}
\end{aligned}
$$

where $\theta$ is the polar angle, and $\Gamma$ is the value of the circulation that satisfied the Kutta condition. Because $\Gamma$ evolved as part of the solution, it is reasonable to be suspicious of this boundary condition as a possible cause of the anomaly. To check this possibility, calculations were made with the "wind-tunnel" code described in reference 12 . In this code, the far-field boundary condition corresponds to no flow through the tunnel walls; that is,

$$
\left.\frac{\partial \phi_{F}}{\partial y}\right|_{y= \pm L}=0
$$

Essentially "free-air" solutions are generated with $L=$ $50 \mathrm{c}$. The multiple solutions were also found with this code.

3. The same anomalous behavior was found with the conservative, Cartesian coordinate code described in reference 13 .

4. No multiple solutions were found with the nonconservative code described in reference 14 (FLO 12).

Although the above tests are not conclusive, they make a strong case for the argument that the problem exists at the differential equation level of the conservative potential formulation. Therefore, with no evidence to the contrary, we will proceed under that assumption.

An interesting feature of the results presented in reference 3 was the occurrence of a "gap" in the lift curve. (See fig. 1.) This gap was explained in reference 3 in terms of a hysteresis effect. In the present investigation, it was determined that the gap comes about because the lift becomes a multivalued function of the angle of attack in this range. However, since the angle of attack remains a single-valued function of the lift, the problem can be made well posed by prescribing the lift and letting the numerical solution determine the angle of attack which satisfies the Kutta condition. (A similar technique was successfully applied by the principal author to another nonunique problem, ref. 15.) In this manner, the complete lift curve for an NACA 0012 airfoil has been evaluated at $M_{\infty}=0.83$ with FLO 36, a modified version of the code described in reference 3 . The results are shown in figure 2 .

For the NACA 0012 airfoil, the results presented in figure 2 show the anomaly occurring in the neighborhood of the zero angle of attack. It should not be inferred from this that the anomaly occurs only at this Mach number and narrow range of angles of attack. At any given Mach number in the transonic regime, a range of angles of attack can be found where the nonuniqueness occurs. For example, for the NACA 0012 airfoil at $M_{\infty}=0.79$, it appears at around $\alpha=1^{\circ}$; at $M_{\infty}=0.60$, it appears at around $\alpha=9^{\circ}$.

It has been speculated that the anomaly is a result of an interaction between an upper-surface shock and a lower-surface shock. However, the anomaly has been observed in a number of cases in which only a single shock (either on the lower or upper surface) is present. A case in point is the flow past an RAE 2822 profile at $M_{\infty}=0.75$ and $\alpha=1^{\circ}$. The lift curve for these conditions is shown in figure 3 . The single shock wave occurs on the upper surface, as shown in figure 4.

Nonuniqueness is not new to potential theory. An incompressible flow past a lifting airfoil with a sharp trailing edge has a one-parameter family of solutions. In this case, the relevant physical solution is singled out by the Kutta condition. At supercritical speeds, solutions with "expansion" shocks are mathematically valid solutions of the governing differential equation. Expansion shocks are ruled out in favor of the physically relevant compression shock by invoking the Second Law of Thermodynamics. In fact, these constraints are now considered integral parts of a numerical algorithm. Why not resolve the present dilemma by imposing some new constraint? If we consider figure 2 again, at zero angle of attack, the symmetric solution corresponding to zero lift seems to be the solution that we would want to single out as relevant with the new constraint. However, 
consider what happens if the angle of attack is increased by a small amount. Which of these solutions is physically relevant? The two with the largest absolute levels of lift can be ruled out on the grounds that they require a discontinuous behavior as $\alpha$ goes to $0^{\circ}$. However, the remaining solution does not seem physically relevant either, since it predicts a negative lift-curve slope. The nonuniqueness problem we are facing is unique in that none of the solutions available seem to be physically relevant! Indeed, if a new constraint is found, its role will not be to single out one of the three solutions presently available, but to find a new solution.

The question now is, Is this anomaly a problem of the conservative potential approximation in the transonic range or a problem of the inviscid flow? To investigate this question, we propose to do a systematic search with an Euler flow code. However, before proceeding to this step, it is necessary to establish the validity of the numerical results of the Euler code. This is the next step in our investigation.

\section{Improvements and Validation of FLO 52-S}

The finite-volume, four-step Runge-Kutta code described in reference 5 (FLO 52) was chosen for this study because of its fast convergence rate. The code solves the time-dependent continuity, $x$-momentum, $y$ momentum, and energy equations in conservative form. To prevent typical central-difference, odd-even oscillations and to capture shock waves, a blended fourth- and second-order explicit artificial damping term is added to the equations. To accelerate the rate of convergence, each mesh cell is advanced at its own local time step, $\Delta t$, and the governing equations are modified by an enthalpy damping term. The calculations were done with a nearly orthogonal "O" mesh with an inverse radial transformation to cluster mesh points near the airfoil. A detailed account of the discretization, damping, and acceleration technique is given in reference 5.

In its original version, the code consistently underpredicted the lift coefficient from potential calculations by as much as 10 percent for lifting subcritical flows. In addition, isomach plots revealed a disturbing behavior near the airfoil surface. As the isomach lines approached the surface, they turned abruptly. This turning indicated an incorrect "boundary-layerlike" behavior. This was also evident in flow-field plots of entropy and total pressure losses.

The cause of the problem was found to be associated with the evaluation of the damping terms near the airfoil surface and in the far field. As described in reference 5, the damping term is made up of contributions from the two coordinate directions $X$ and $Y$ with associated indexes $i$ and $j$, respectively. Let $X$ be the coordinate along a ring of the " $\mathrm{O}$ " mesh and $Y$ be the coordinate in the normal direction. The damping term is given by

$$
\begin{aligned}
D w & =D_{X} w+D_{Y} w \\
D_{X} w & =d_{i+1 / 2, j}-d_{i-1 / 2, j} \\
D_{Y} w & =d_{i, j+1 / 2}-d_{i, j-1 / 2}
\end{aligned}
$$

where $w$ is the vector of conservative unknowns, and a typical $d$ term is defined by

$$
\begin{aligned}
d_{i, j+1 / 2}= & \frac{h_{i, j+1 / 2}}{\Delta t}\left[\varepsilon_{i, j+1 / 2}^{(2)} \Delta w_{i, j+1 / 2}\right. \\
& -\varepsilon_{i, j+1 / 2}^{(4)}\left(\Delta w_{i, j+3 / 2}-2 \Delta w_{i, j+1 / 2}\right. \\
& \left.\left.+\Delta w_{i, j-1 / 2}\right)\right]
\end{aligned}
$$

The symbol $h$ represents the cell area, $\varepsilon^{(2)}$ and $\varepsilon^{(4)}$ are coefficients, defined in reference 5 , which depend on the second derivative of pressure, and

$$
\Delta w_{i, j+1 / 2}=w_{i, j+1}-w_{i, j}
$$

The evaluation of the $d$ terms presents some problems near the airfoil surface, $j \leq 2$, and near the last ring of the "O" mesh, $j \geq J_{\max }-1$, because of the lack of information near these cells. In the original code, $\Delta w$ terms inside the airfoil and outside the last ring of the "O" mesh were constructed with a third-order extrapolation from inside the flow field.

The "boundary-layerlike" behavior was eliminated by the following procedure. At a ghost point $j=$ 0 inside the airfoil, the pressure, density, and total enthalpy were obtained by linear extrapolation from the flow field. The velocity components at the cell center immediately next to the airfoil surface were decomposed into components normal and tangent to the surface. These were then reflected to obtain components at the ghost cell. With this information, it was possible to evaluate

$$
\Delta w_{i, 1 / 2}=w_{i, 1}-w_{i, o}
$$

The missing term $\Delta w_{i,-1 / 2}$ was evaluated by equating it to $\Delta w_{i, 1 / 2}$. Although this procedure eliminated the "boundary-layerlike" behavior, it had little effect on the low-lift level being predicted. Surprisingly, the problem with the lift was corrected by modifying the evaluation of the far-field damping terms. The finitevolume integration is performed only up to $J_{\max }-1$; values of $w$ at $J_{\max }$ are obtained by satisfying the far-field boundary conditions, which use no damping. Therefore, to evaluate the damping terms at $J_{\max }-1$, only additional information for the term $\Delta w_{i, J_{\max }+1 / 2}$ is required. When this term was approximated by

$$
\Delta w_{i, J_{\max }+1 / 2}=\Delta w_{i, J_{\max }-1 / 2}
$$

the error in the lift level was reduced to about 1 percent. 
The far-field boundary condition evaluation in the original code used the method of Rudy and Strikwerda (ref. 16), which introduces an additional free parameter into the calculation. This method was replaced by the following procedure, which proved more robust and made a slight improvement in the convergence rate. At a subsonic point, a frame of reference perpendicular and tangential to the last ring of the " $O$ " mesh is constructed. In the plane made by the perpendicular to the ring of the " $\mathrm{O}$ " mesh and the time axis, the Riemann "invariant" that propagates along the characteristic coming into the computational region is prescribed from known free-stream values. The Riemann "invariant" propagated by the outgoing characteristic is extrapolated from computed values. From these two quantities, the speed of sound and the velocity component normal to the ring of the "O" mesh are determined. If the point corresponds to an inflow point, the entropy and velocity components tangent to the ring of the "O" mesh are prescribed from free-stream values. If the point corresponds to an outflow point, the entropy and velocity components tangent to the ring of the "O" mesh are extrapolated from computed values. From these four quantities, the conservative unknown vector $w$ is evaluated. For supersonic inflow, all quantities are prescribed, but for supersonic outflow, all quantities are extrapolated from computed values.

The code was also modified to include, as an option, the effect of a far-field vortex. To this end, the circulation is approximated from the lift calculated by the integration of the surface pressure. Then, the free-stream Cartesian velocity components modified to account for the vortex are defined as

$$
\begin{aligned}
& \bar{u}_{\infty}=u_{\infty}-\kappa \sin \theta \\
& \bar{v}_{\infty}=v_{\mathrm{co}}+\kappa \cos \theta
\end{aligned}
$$

where

$$
\begin{aligned}
\kappa & =\frac{\beta \Gamma}{2 \pi r\left[1-M_{\infty}^{2} \sin ^{2}(\theta-\alpha)\right]} \\
\Gamma & =-\frac{1}{2} c a_{\infty} M_{\infty} c_{l}
\end{aligned}
$$

and $r, \theta$ are polar coordinates in the physical plane with the origin at the center of the airfoil. The free-stream thermodynamic variables are recomputed by using the magnitude of the modified free-stream velocity given by equations (11) and (12), the steady-state Bernoulli equation, and the known value of entropy at the point in question. In general, the far-field vortex had very little effect on the calculated results. This is probably the result of the location of the last ring of the "O" mesh, which in these calculations corresponded to about 100 chords from the airfoil.

The last major modification to the Euler code con- sisted of a second-order accurate integration of the normal pressure gradient at the surface of the airfoil to evaluate the surface pressure. As discussed in reference 5, because of the finite-volume formulation and the fact that the fluxes convected across the airfoil surface are zero, only the pressure needs to be evaluated at the surface. In this connection, it should be stressed that the evaluation of ghost points, discussed in relation to the damping terms, is not related to the evaluation of the surface boundary conditions.

For reference purposes, we will designate the modified Euler code as FLO 52-S. A large number of lifting subcritical cases were calculated with this code by using a $120 \times 34$ mesh and with the potential code FLO 36 by using a $192 \times 32$ mesh. Results are presented for one of the "most difficult" subcritical lifting cases computed - an NACA 0012 airfoil at $M_{\infty}=0.30$ and $\alpha=10^{\circ}$. A partial view of the mesh corresponding to $120 \times 34$ points is shown in figure 5 . Figure 6 shows that second-order accuracy in the lift and drag coefficients is attainable with mesh refinement. Figure 7 shows a typical convergence behavior. The residuals plotted in figure $7(\mathrm{a})$ are the root-mean-square deviations of $\Delta \rho / \Delta t, \Delta \rho u / \Delta t$, and $\Delta \rho v / \Delta t$ evaluated over the entire field. Figure $7(\mathrm{~b})$ shows the convergence of lift, drag, and moment coefficients, each evaluated from the integration of the surface pressure. A comparison between FLO 36 and FLO 52-S for the surface pressure distribution, isomach contours, and streamline trajectories is shown in figures 8 to 10 . Practically no difference can be observed if the results of the two calculations are overlaid; we emphasize the results presented correspond to a case that showed the most disagreement with a potential calculation.

At supercritical speeds, the potential calculations are only approximate solutions to the inviscid flow. Typically, these calculations have a stronger shock wave, which appears further aft than in Euler calculations. Two typical results at $M_{\infty}=0.82$ and 0.86 (both at zero angle of attack) are shown in figures 11 and 12. A check on how well the shock wave is captured is shown in figure 13, where the calculated shock jump is compared with the exact jump. Taking into consideration the difficulty in reading the results because of the shock-wave smearing and the Zierep singularity, the agreement is quite good. For comparison, the results of the nonconservative potential code, FLO 12, are also included.

\section{Nonuniqueness Study}

The lift curve shown in figure 2 is repeated in figure 14, along with the results from the Euler calculation (FLO 52-S) and the nonconservative potential code FLO 12. As can be seen from this figure, the Euler results do not show any anomaly at this particular 
Mach number. Can it be that the Euler solution will be anomalous at some other Mach number?

In order to study this, consider the angle made by the lift curve with the abscissa at zero lift. If this angle exceeds $90^{\circ}$, then we are into the anomalous region, since a positive angle of attack produces negative lift. Figure 15 shows the behavior of this angle as a function of free-stream Mach number for conservative potential, nonconservative potential, and Euler calculations. The figure shows that the conservative potential solution becomes progressively worse as the free-stream Mach number increases and eventually crosses over into the multiple solution region at about $M_{\infty}=0.82$. Both Euler and nonconservative potential solutions remain well-behaved throughout the Mach number range. The anomaly, therefore, is indicative of a breakdown of the conservative potential formulation, not of inviscid theory, per se.

In figure 16, the plot of figure 15 is repeated in terms of transonic similarity parameters (ref. 17). In addition to the results obtained for an NACA 0012 airfoil, results for an NACA 0006 airfoil are also shown in this figure. It is interesting to see that the conservative potential results appear to collapse into a single curve in the similarity plane. This is another indication that the numerical code is predicting the behavior expected of the partial differential equation. It furthermore indicates that the nonuniqueness problem will occur even for very weak shock waves if the airfoil thickness $\tau$ is made sufficiently small. The fact that the Euler results do not collapse into a single curve in the similarity plane is not unexpected, since the similarity law is only valid for the small-disturbance potential equation. The rapid drop in $\partial c_{l} / \partial \alpha$ shown in figure 16 for $\chi<0.8$ implies a drop in lift, which has been explained as an inviscid phenomenon associated with the inefficient production of lift that occurs when the upper surface shock wave moves to the trailing edge (ref. 17 and ref. 18, p. 656).

Classically, potential theory is accepted as a good approximation to the rotational inviscid flow in the transonic range if the shock is weak. The shock strength is measured by $M_{s}^{2}-1$, where $M_{s}$ is the Mach number upstream of the shock. The rationale is based on the fact that the entropy produced at the shock is proportional to the cube of the shock strength. Thus, the potential approximation is accepted if

$$
\left(M_{s}^{2}-1\right)^{3} \ll 1
$$

In practical applications, $M_{s}$ is required to be less than 1.3. However, the results of figure 16 are not consistent with this requirement. Two potential flows are topologically equivalent for affine airfoil profiles if they have the same transonic similarity parameter $\chi$. For example, flow past an NACA 0012 airfoil at
$M_{\infty}=0.820, \alpha=0^{\circ}$, and flow past an NACA 0006 airfoil at $M_{\infty}=0.879, \alpha=0^{\circ}$ both correspond to the same value of the transonic similarity parameter $\chi$. This value corresponds to conditions on the verge of nonuniqueness. It is reasonable to expect that if for one of these flow fields the potential assumption ceases to be valid, then it should cease to be valid for the other flow field as well. The computed surface Mach numbers for these two cases are illustrated in figures 17 and 18. It is evident from figure 18 that the thinner airfoil does not violate the classical criterion. This should be expected, since relation (15) is not scaled according to similarity rules. The problem can be overcome if we do not allow the similarity-scaled entropy

$$
S \approx \frac{\left(M_{s}^{2}-1\right)^{3}}{\tau^{2}}
$$

to be greater than the value it reaches when the nonuniqueness occurs. For example, for an NACA 0012 airfoil near $\alpha=0^{\circ}$, nonuniqueness is observed at a value of $M_{s}$ of approximately 1.3 ; with this information, we can compute from relation (16) a value of $S$ which we will designate $S_{o}$. For any other affine profile of thickness $\tau$, we must require that the Mach number at the shock obey the constraint

$$
M_{s}<\left(1+S_{o}^{1 / 3} \tau^{2 / 3}\right)^{1 / 2}
$$

For thinner airfoils, this constraint severely limits the transonic region for which the potential theory represents a valid approximation to an inviscid rotational flow. How to extend the constraint given by relation (17) or develop some other rule that will prevent the application of the conservative potential formulation in the anomalous range for airfoils of different families is the subject of an ongoing effort.

The conservative potential model develops some problems when applied to quasi-one-dimensional nozzle flows. It is believed that a heuristic explanation of the anomaly can be reached by examining this problem. With the nozzle choked, two isentropic solutions are possible-one corresponding to subsonic flow, the other corresponding to supersonic flow. Of these two, the one that appears is determined by matching the downstream imposed pressure. If a downstream value of pressure is prescribed between these two extremes, a shock wave forms in the divergent part of the nozzle or outside the nozzle. The position of the shock is established by matching the prescribed downstream pressure. The above description is properly modeled by the Euler equations. What happens if the problem is described by the conservative potential equation? If the downstream value of pressure corresponds to the subsonic isentropic solution, a solution with supersonic flow downstream of the throat followed by an isentropic com- 
pression shock is still possible, since this solution will satisfy the downstream boundary condition. Moreover, the shock can be placed anywhere downstream of the throat and still satisfy the differential equation and the boundary condition. In other words, there is an uncertainty with regard to the shock position. The problem comes about because the exact isentropic jump is being satisfied and this jump connects the subsonic and supersonic isentropic branches. This is not the case with the Euler equations because of the entropy produced at the shock, or with the nonconservative potential formulation because the exact isentropic jump is not satisfied. It is believed that this inherent weakness of the conservative formulation to fix the shock position in the quasi-one-dimensional problem is also responsible for the anomaly observed in two dimensions.

It can be argued, however, that the downstream far-field boundary condition imposed on the twodimensional airfoil problem is specified in terms of $\phi$, not $\phi_{x}$ (specifying the back pressure is equivalent to specifying $\phi_{x}$ ), and that by specifying $\phi$ the problem should then be well posed. However, this argument fails to recognize that the shock-wave position is more sensitive to the pressure level that develops at the trailing edge than to the pressure level on the downstream far field. Thus, the analogy with the quasi-one-dimensional problems remains valid. It is interesting that similar ideas for internal flow problems were independently developed by Deconinck and Hirsch in a recent paper (ref. 19).

If the nonuniqueness is indeed a problem in the shock jump representation, then it could be resolved by abandoning the isentropic, mass-conserving shock-wave formulation for some other shock representation. (See, for example, ref. 20.) However, it seems that to retain a rational formulation, some form of shock-fitting will be required.

\section{Concluding Remarks}

The nonuniqueness problem occurring at transonic speeds with the conservative potential equation has been investigated numerically. Additional evidence has been provided which supports the thesis that this nonuniqueness is a problem inherent in the conservative potential differential equation rather than a consequence of the finite-difference approximation. The hysteresis effect previously observed has been shown to be a numerical result of the multivalue nature of the computed lift in a certain range of angles of attack at a given free-stream Mach number. Since none of the multiple solutions obtained with the conservative potential formulation seem relevant to the physical problem, a breakdown of the theory is indicated. It appears that in order to avoid this anomaly, the present conservative formulation must be abandoned. A more restrictive criterion for the validity of potential theory in the transonic range has been proposed. This criterion shows that shock-wave strength should be measured relative to airfoil thickness. The numerical results obtained with the Euler code indicate that this nonuniqueness problem is not inherent in the inviscid solution but results from the approximate treatment of shock waves inherent in the conservative potential model. However, it is felt that more research is necessary to settle this issue conclusively.

\section{Langley Research Center}

National Aeronautics and Space Administration

Hampton, VA 23665

October 18, 1984

\section{References}

1. Lax, Peter D.: Weak Solutions of Nonlinear Hyperbolic Equations and Their Numerical Computation. Commun. Pure \& Appl. Math., vol. VII, no. 1, Feb. 1954, pp. 159-193.

2. Bers, Lipman: Existence and Uniqueness of a Subsonic Flow Past a Given Profile. Commun. Pure \& Appl. Math., vol. VII, no. 3, Aug. 1954, pp. 441-504.

3. Steinhoff, John; and Jameson, Antony: Multiple Solutions of the Transonic Potential Flow Equation. A Collection of Technical Papers-AIAA 5th Computational Fluid Dynamics Conference, June 1981, pp. 347-353. (Available as AIAA-81-1019.)

4. Nixon, David, ed.: Transonic Aerodynamics. American Inst. Aeronaut. \& Astronaut., Inc., c.1982.

5. Jameson, A.; Schmidt, Wolfgang; and Turkel, Eli: Numerical Solution of the Euler Equations by Finite Volume Methods Using Runge Kutta Time Stepping Schemes. AIAA-81-1259, June 1981.

6. Salas, M. D.; Gumbert, C. R.; and Turkel, E.: Nonunique Solutions to the Transonic Potential Flow Equation. AIAA J., vol. 22, no. 1, Jan. 1984, pp. 145-146.

7. Salas, M. D.; Jameson, A.; Melnik, R. E.: A Comparative Study of the Nonuniqueness Problem of the Potential Equation. A Collection of Technical PapersAIAA 6th Computational Fluid Dynamics Conference, July 1983, pp. 48-60. (Available as AIAA-83-1888.)

8. Jameson, Antony: Acceleration of Transonic Potential Flow Calculations on Arbitrary Meshes by the Multiple Grid Method. A Collection of Technical Papers-AIAA Computational Fluid Dynamics Conference, July 1979, pp. 122-146. (Available as AIAA Paper 79-1458.)

9. Ludford, G. S. S.: The Behavior at Infinity of the Potential Function of a Two-Dimensional Subsonic Compressible Flow. J. Math. Phys., vol. XXX, no. 3, Oct. 1951, pp. 117-130.

10. Bauer, Frances; Garabedian, Paul; Korn, David; and Jameson, Antony: Supercritical Wing Sections II. Vol- 
ume 108 of Lecture Notes in Economics and Mathematical Systems, Springer-Verlag, 1975.

11. Dougherty, F. Carroll; Holst, Terry L.; Gundy, Karen L.; and Thomas, Scott D.: TAIR-a Transonic Airfoil Analysis Computer Code. NASA TM-81296, 1981.

12. Doria, Michael L.; and South, Jerry C., Jr.: Transonic Potential Flow and Coordinate Generation for Bodies in a Wind Tunnel. AIAA-82-0223, Jan. 1982.

13. Wedan, Bruce; and South, Jerry C., Jr.; A Method for Solving the Transonic Full-Potential Equation for General Configurations. A Collection of Technical PapersAlAA 6th Computational Fluid Dynamics conference, July 1983, pp. 515-526. (Available as AIAA-83-1889.)

14. Jameson, Antony: Iterative Solution of Transonic Flows Over Airfoils and Wings, Including Flows at Mach 1. Commun. Pure \& Appl. Math., vol. XXVII, no. 3, May 1974, pp. 283-309.

15. Salas, M. D.; and Morgan, B. D.: Stability of Shock Waves Attached to Wedges and Cones. AIAA J., vol. 21, no. 12, Dec. 1983, pp. 1611-1617.
16. Rudy, David H.; and Strikwerda, John C.: A Nonreflecting Outflow Boundary Condition for Subsonic Navier-Stokes Calculations. J. Comput. Phys., vol. 36, no, 1, June 1980, pp. 55-70.

17. Cole, J. D.; Solomon, G. E.; and Willmarth, W. W.: Transonic Flow Past Simple Bodies. J. Aeronaut. Sci., vol. 20, no. 9, Sept. 1953, pp. 627-634.

18. Mair, W. A.; and Beavan, J. A.: Flow Past Aerofoils and Cylinders. Modern Developments in Fluid DynamicsHigh Speed Flow, L. Howarth, ed., Oxford Univ. Press, 1953, pp. 612-687.

19. Deconinck, H.; and Hirsch, Ch.: Boundary Conditions for the Potential Equation in Transonic Internal Flow Calculation. ASME Paper 83-GT-135, Mar. 1983.

20. Klopfer, Goetz H.; and Nixon, David: Non-Isentropic Potential Formulation for Transonic Flows. AIAA-830375, Jan. 1983. 


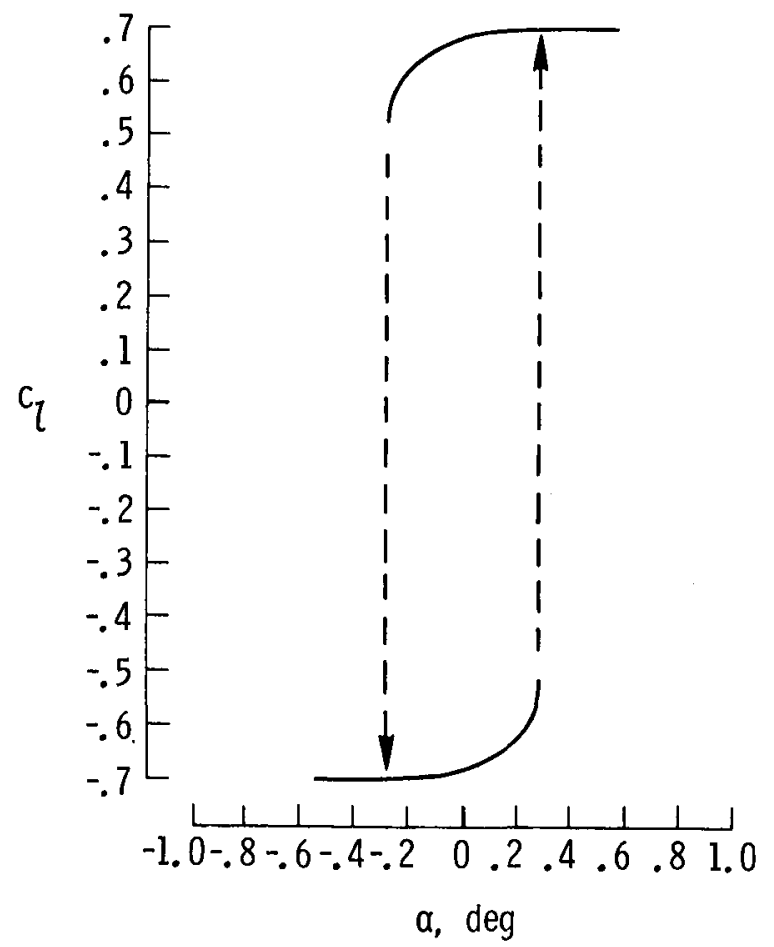

Figure 1. Lift curve obtained with FLO 36 for NACA 0012 airfoil with hysteresis effect as in reference $3 . M_{\infty}=0.83$.

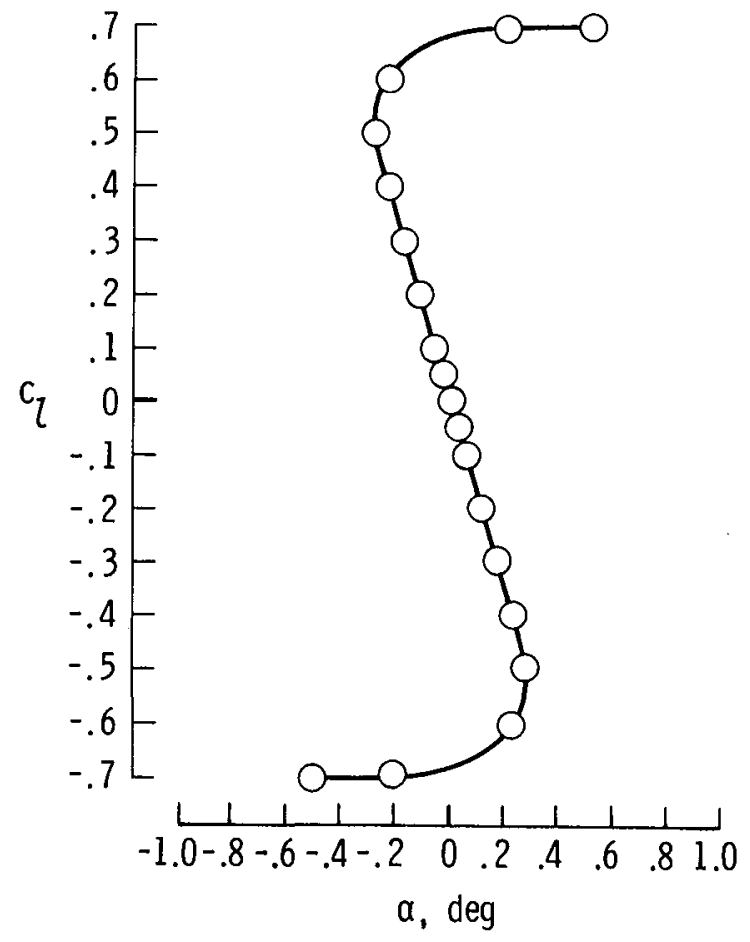

Figure 2. Lift curve obtained with FLO 36 for NACA 0012 airfoil at $M_{\infty}=0.83$.

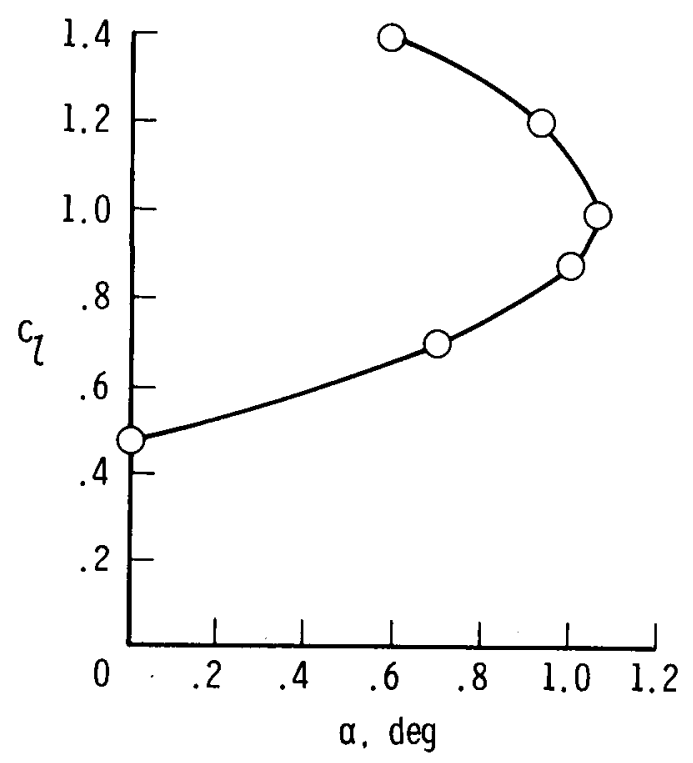

Figure 3. Lift curve obtained with FLO 36 for RAE

2822 airfoil at $M_{\infty}=0.75$. 


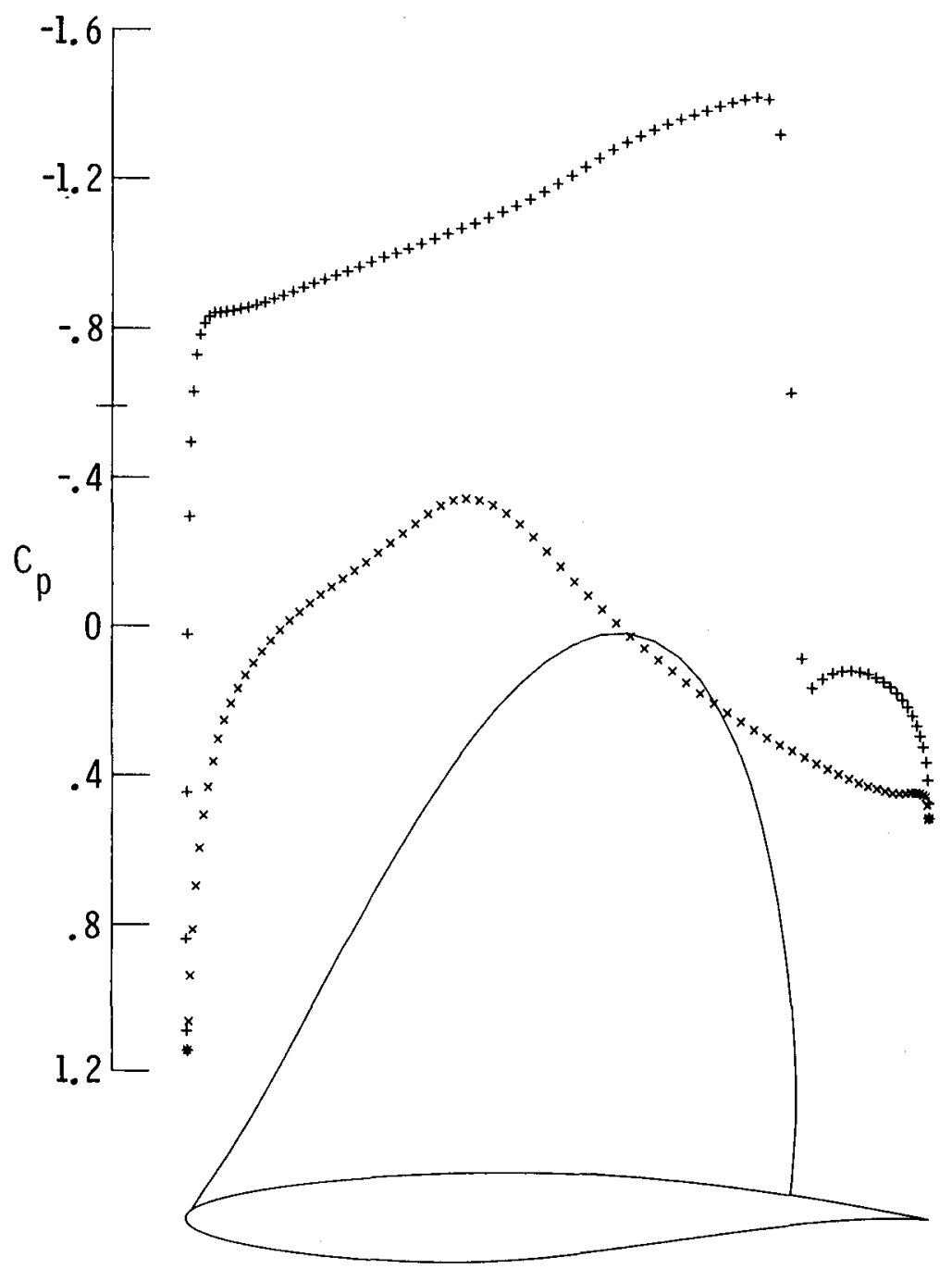

Figure 4. Pressure coefficient computed with FLO 36 for RAE 2822 airfoil at $M_{\infty}=0.75$ and $\alpha=1^{\circ}$ 


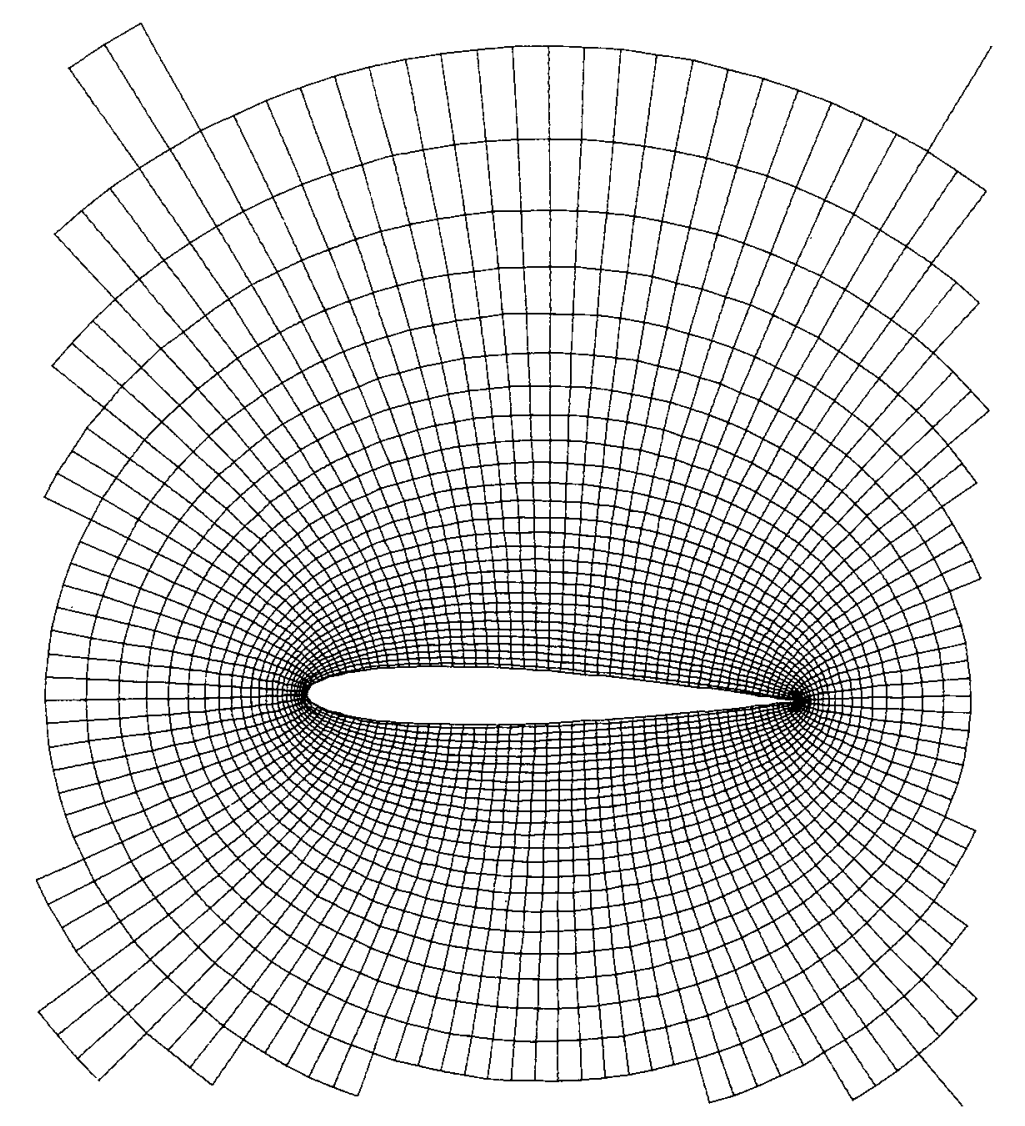

Figure 5. Partial view near airfoil of mesh used in FLO 52-S calculations.

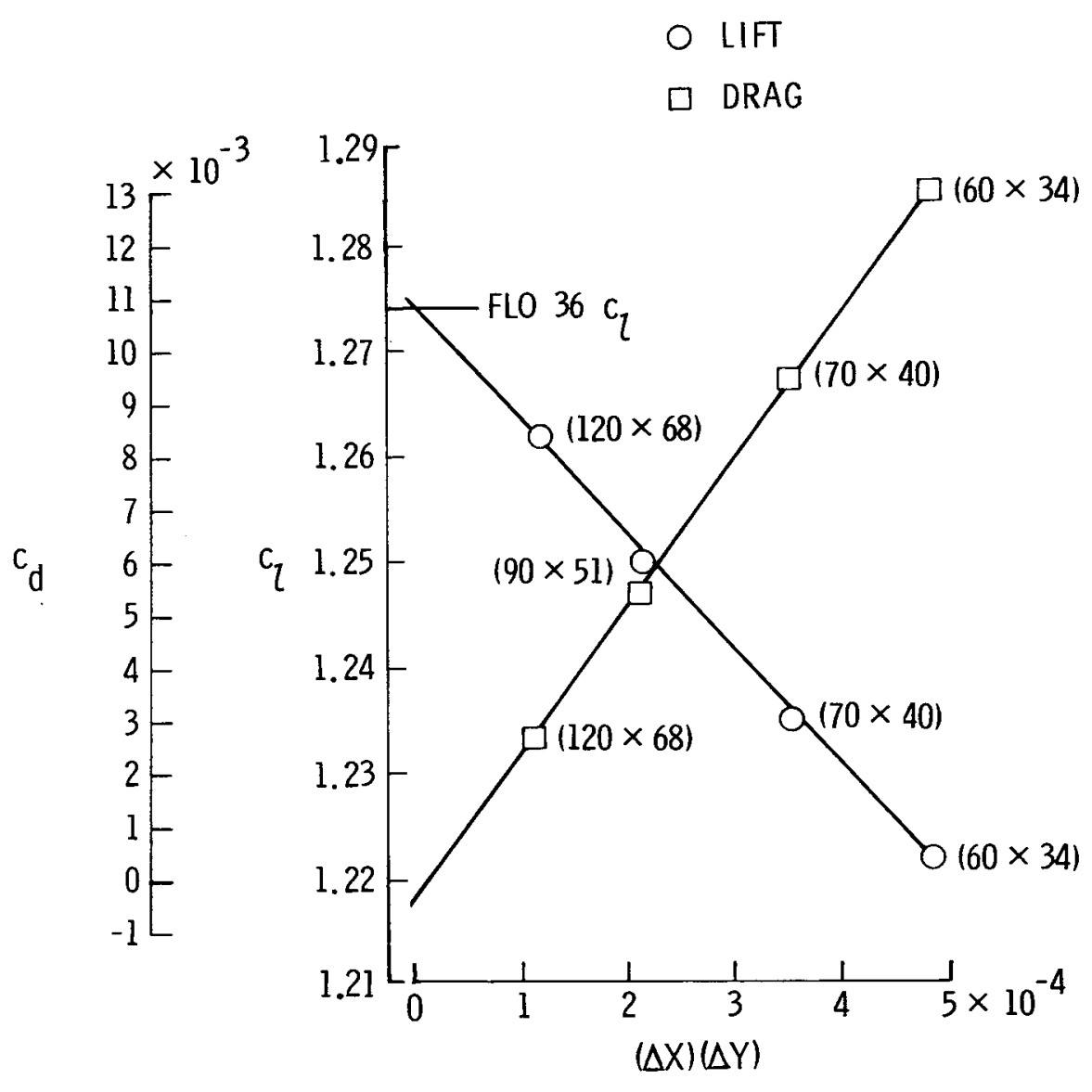

Figure 6. Convergence of lift coefficients (circles) and drag coefficients (squares) with mesh refinement for FLO 52-S. NACA 0012 airfoil; $M_{\infty}=0.30 ; \alpha=10^{\circ}$. 


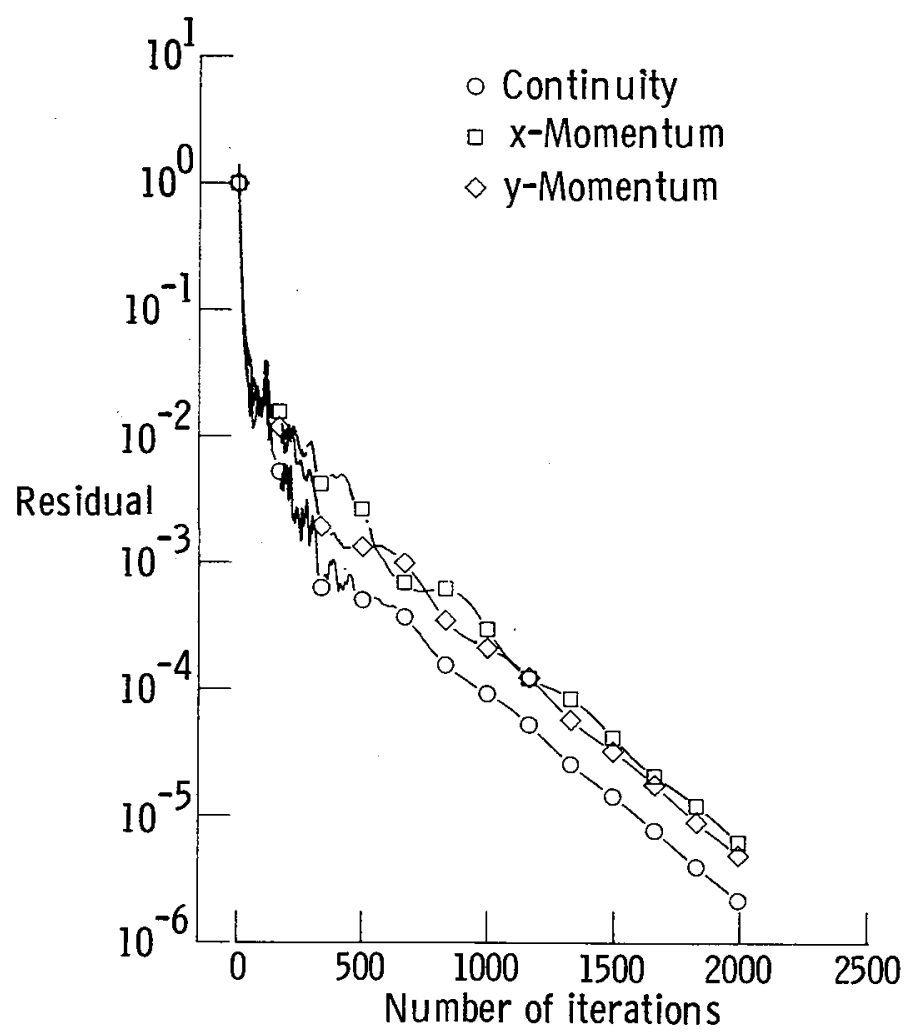

(a) Residual decays.

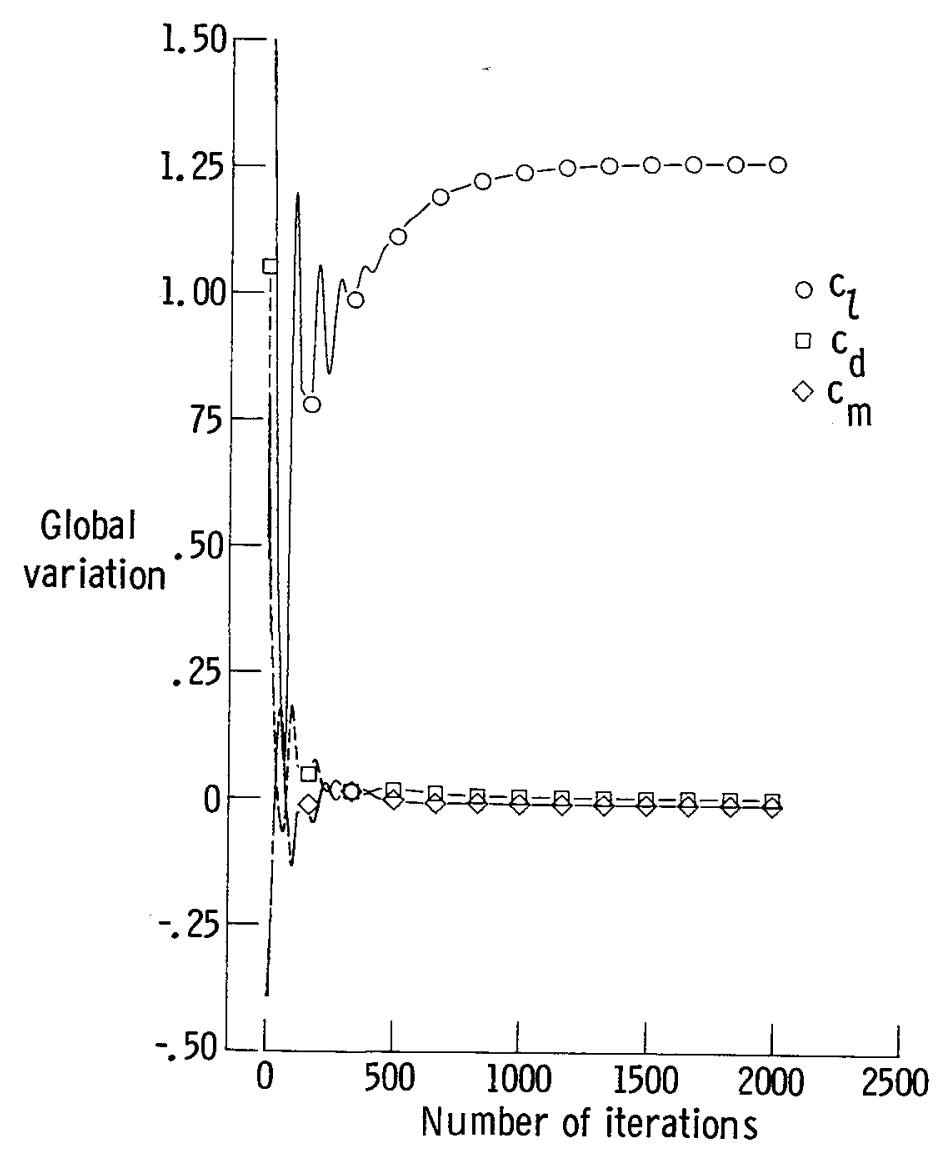

(b) Lift, drag, and pitching-moment coefficients.

Figure 7. Convergence histories. NACA 0012 airfoil; $M_{\infty}=0.30 ; \alpha=10^{\circ} ; 120 \times 34$ mesh. 


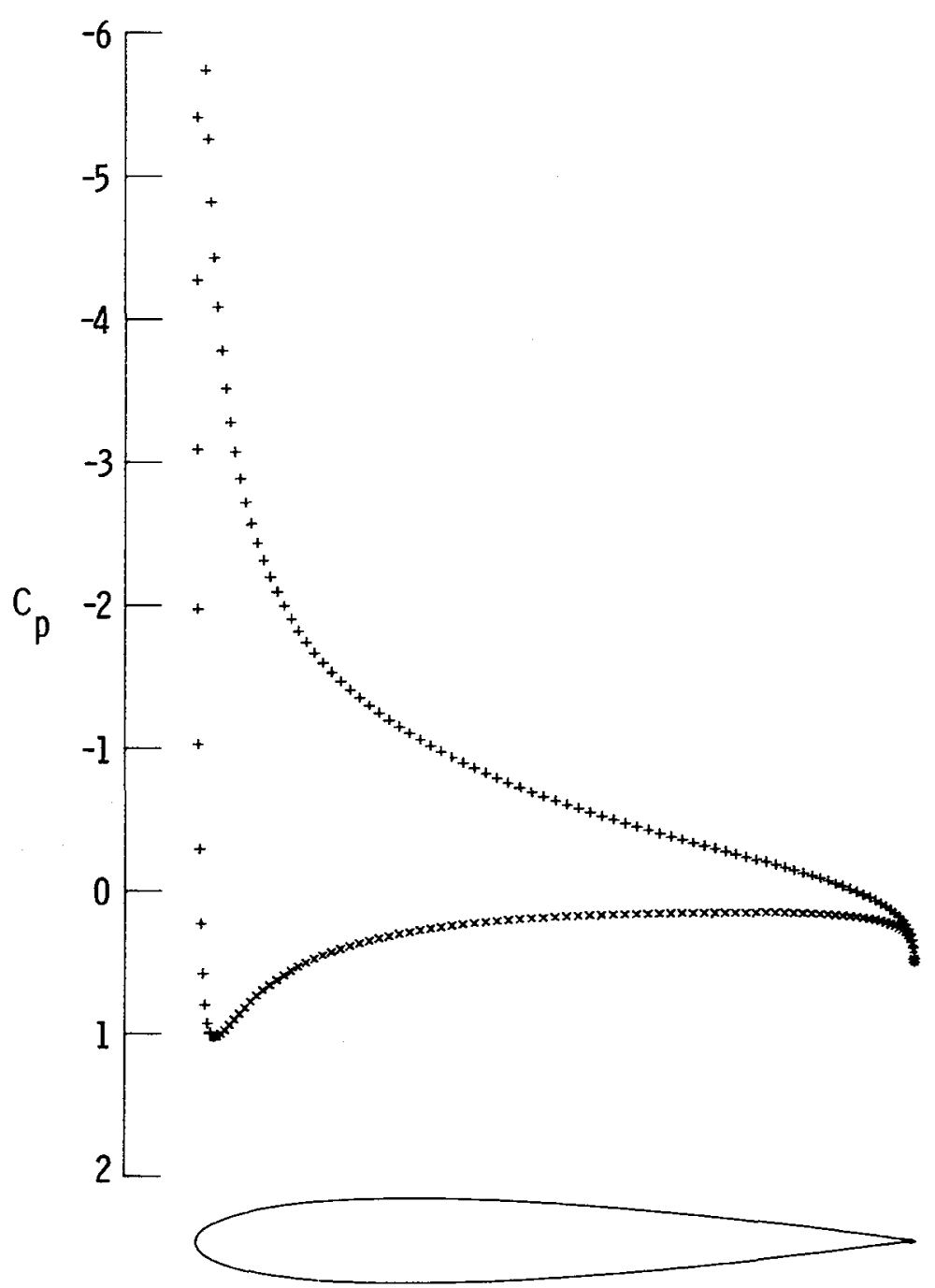

(a) Computed with FLO 36. $c_{l}=1.2733 ; c_{d}=0.0001$ $c_{m}=-0.0100 ; 192 \times 32 \mathrm{mesh} ; 25$ cycles, residual of $0.452 \times 10^{-12}$.

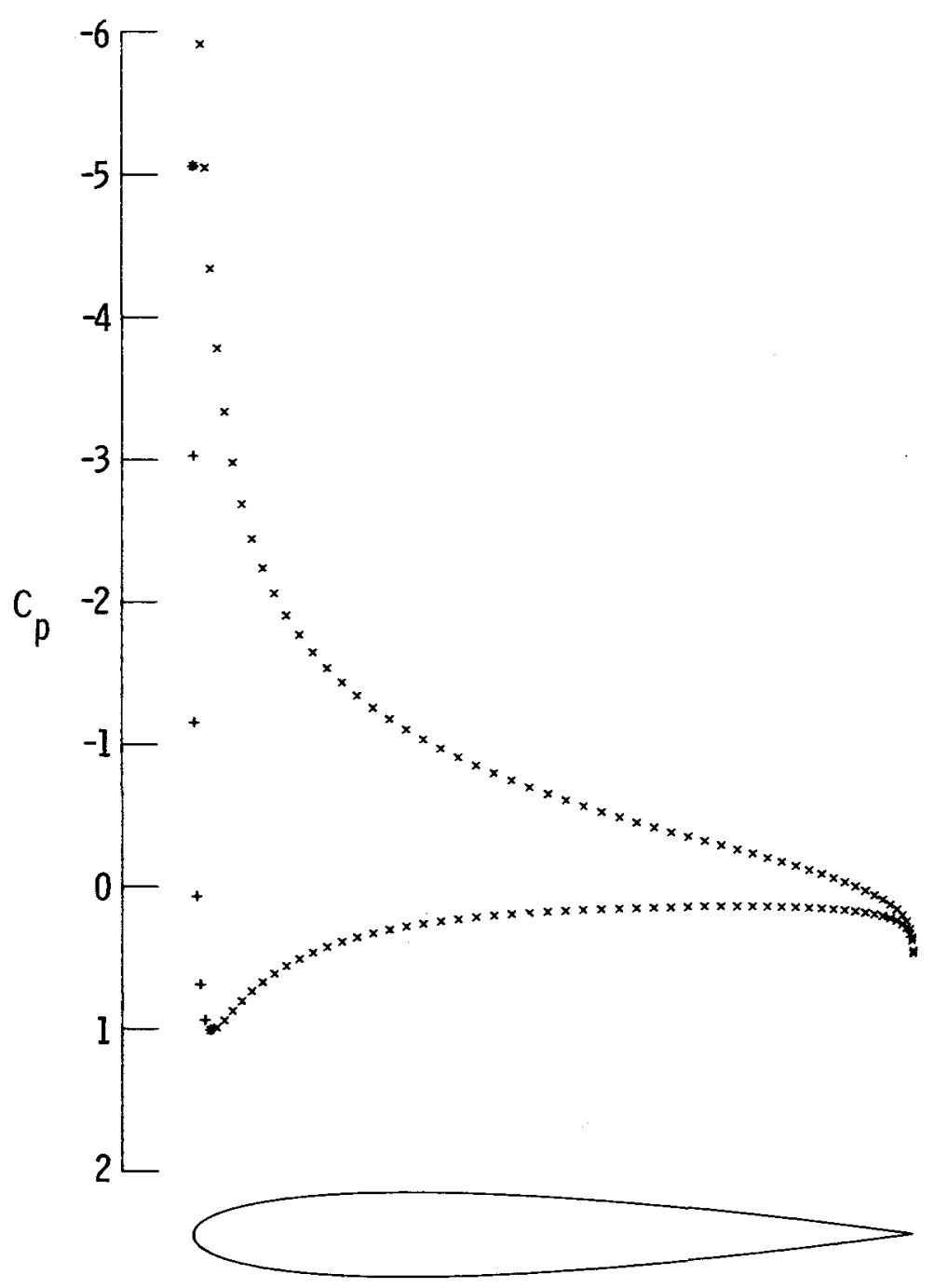

(b) Computed with FLO 52-S. $c_{l}=1.2613 ; c_{d}=0.0026$; $c_{m}=-0.0104 ; 120 \times 34$ mesh, 2000 cycles.

Figure 8. Pressure coefficient for NACA 0012 airfoil at $M_{\infty}=0.30$ and $\alpha=10^{\circ}$. 


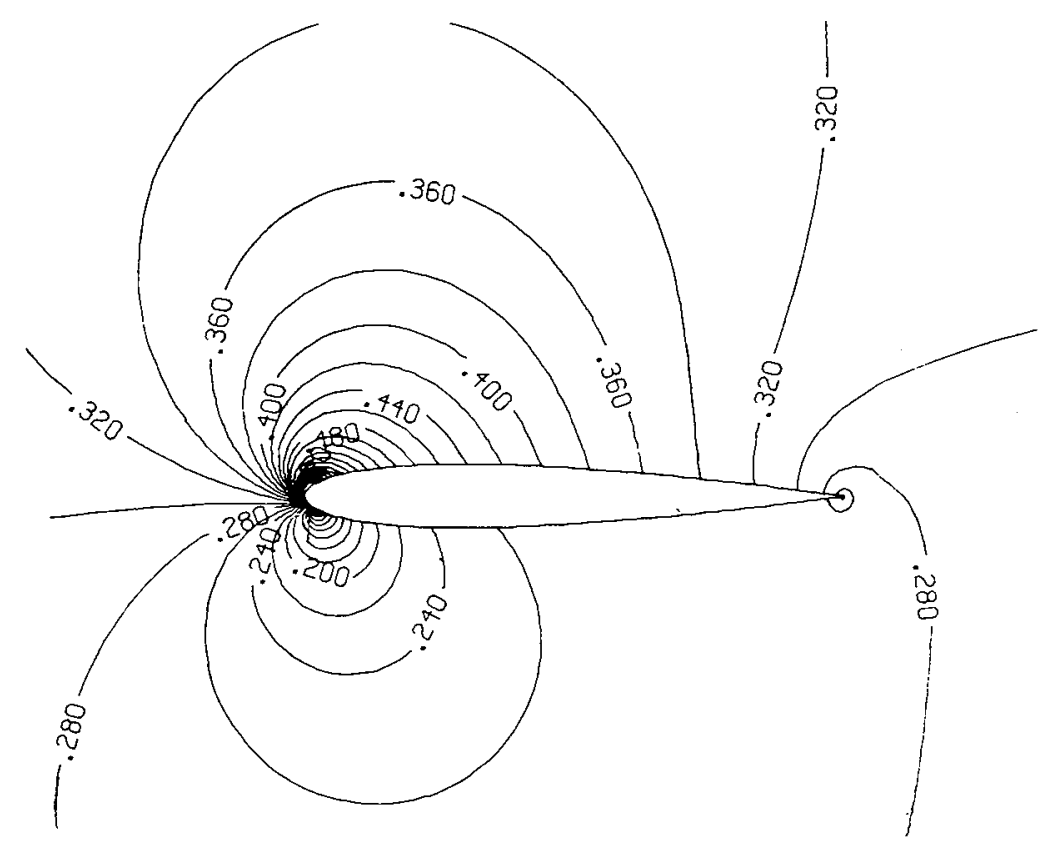

(a) Computed with FLO 36 .

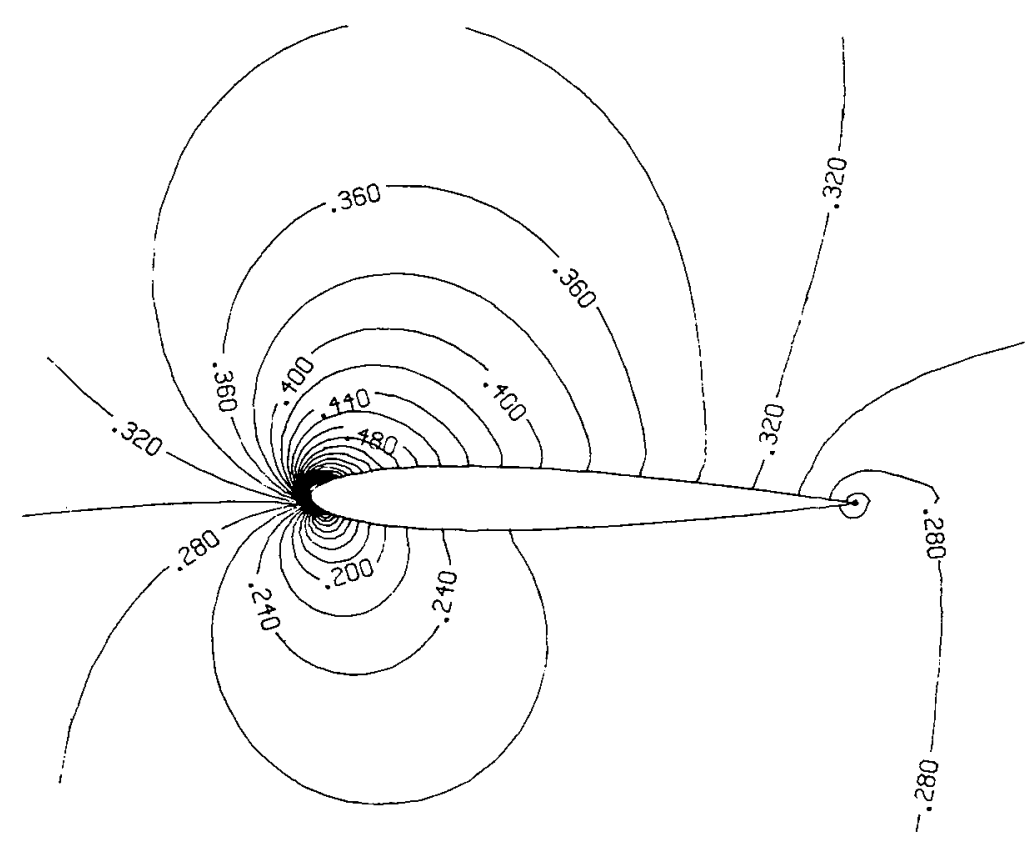

(b) Computed with FLO 52-S.

Figure 9. Mach number contours for NACA 0012 airfoil at $M_{\infty}=0.30$ and $\alpha=10^{\circ}$. 


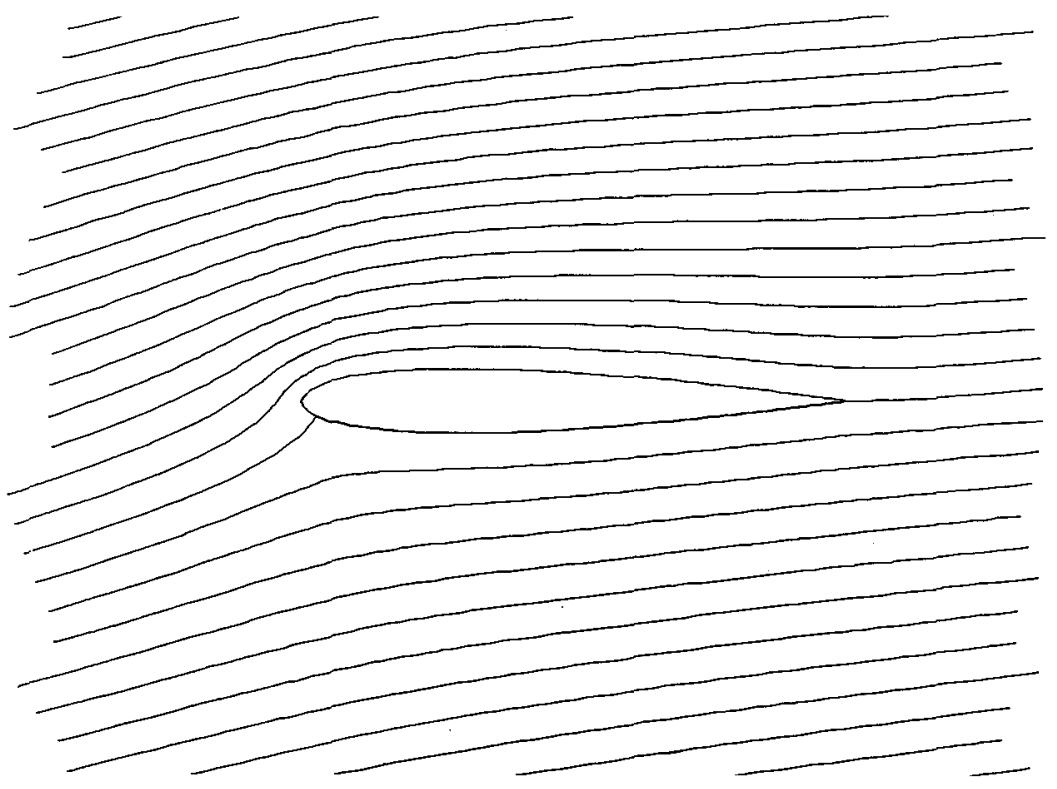

(a) Computed with FLO 36.

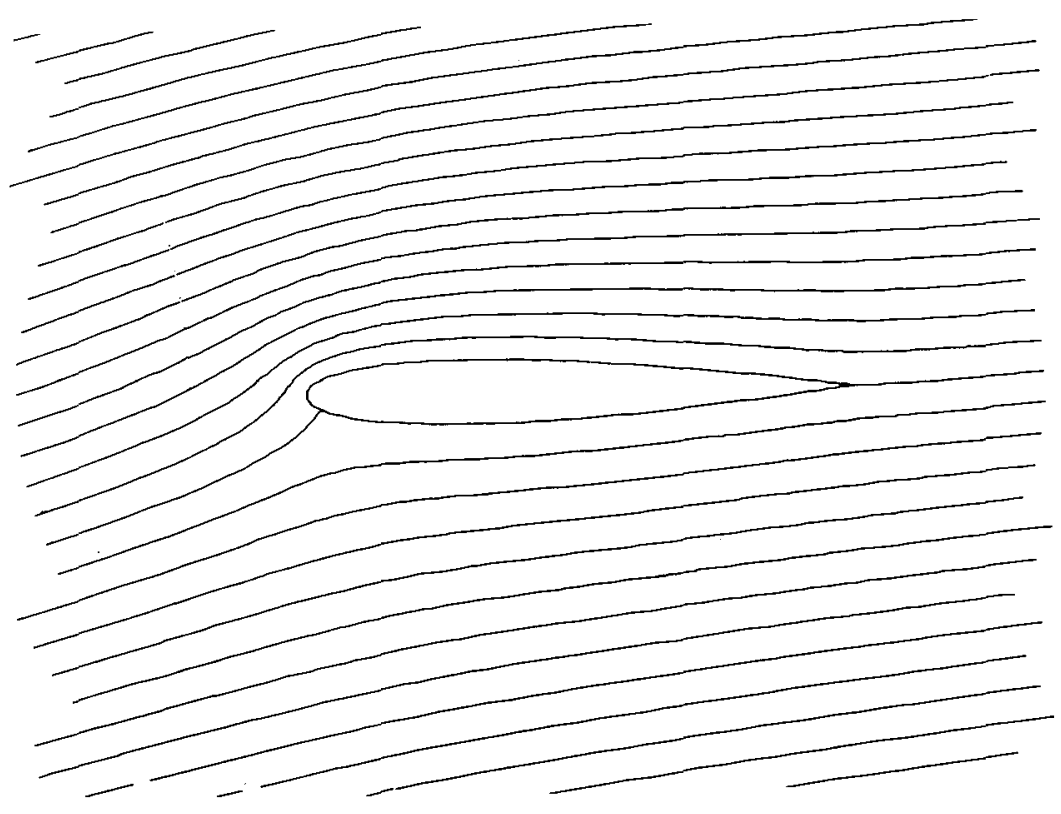

(b) Computed with FLO 52-S.

Figure 10. Streamline pattern for NACA 0012 airfoil at $M_{\infty}=0.30$ and $\alpha=10^{\circ}$. 


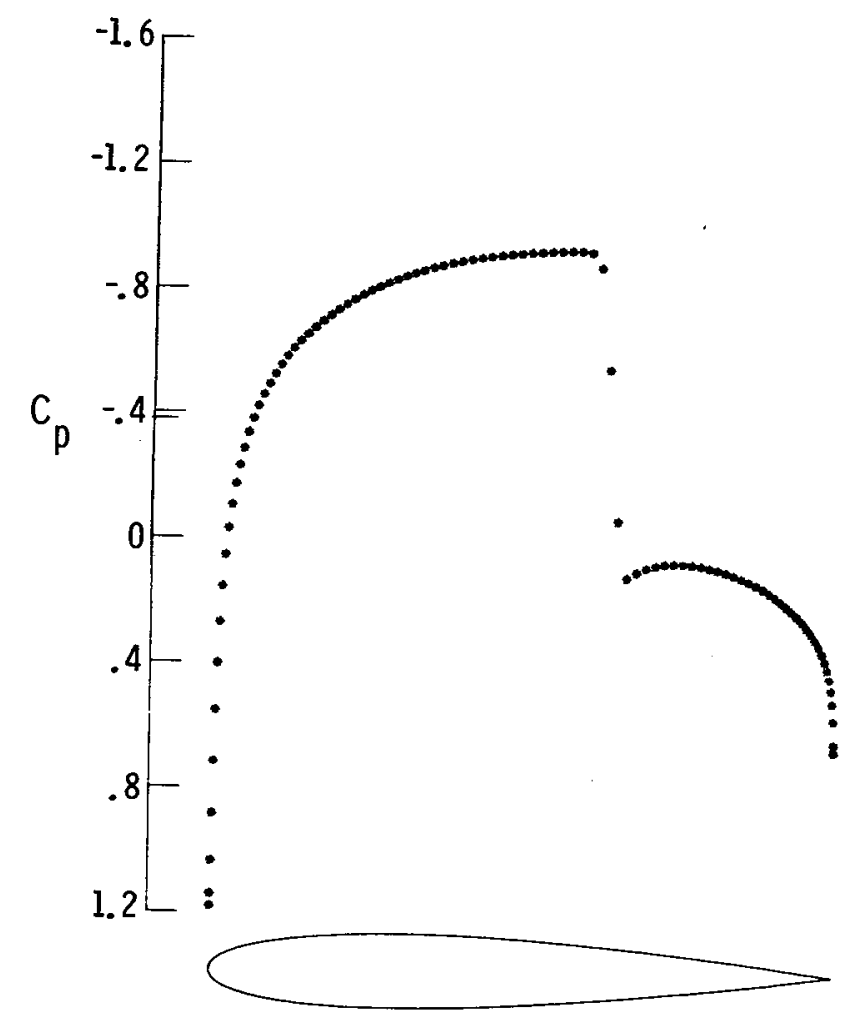

(a) Computed with FLO 36. $c_{l}=0.0000 ; c_{d}=0.0186$; $c_{m}=0.0000 ; 192 \times 32$ mesh; 71 cycles; residual of
$0.660 \times 10^{-12}$

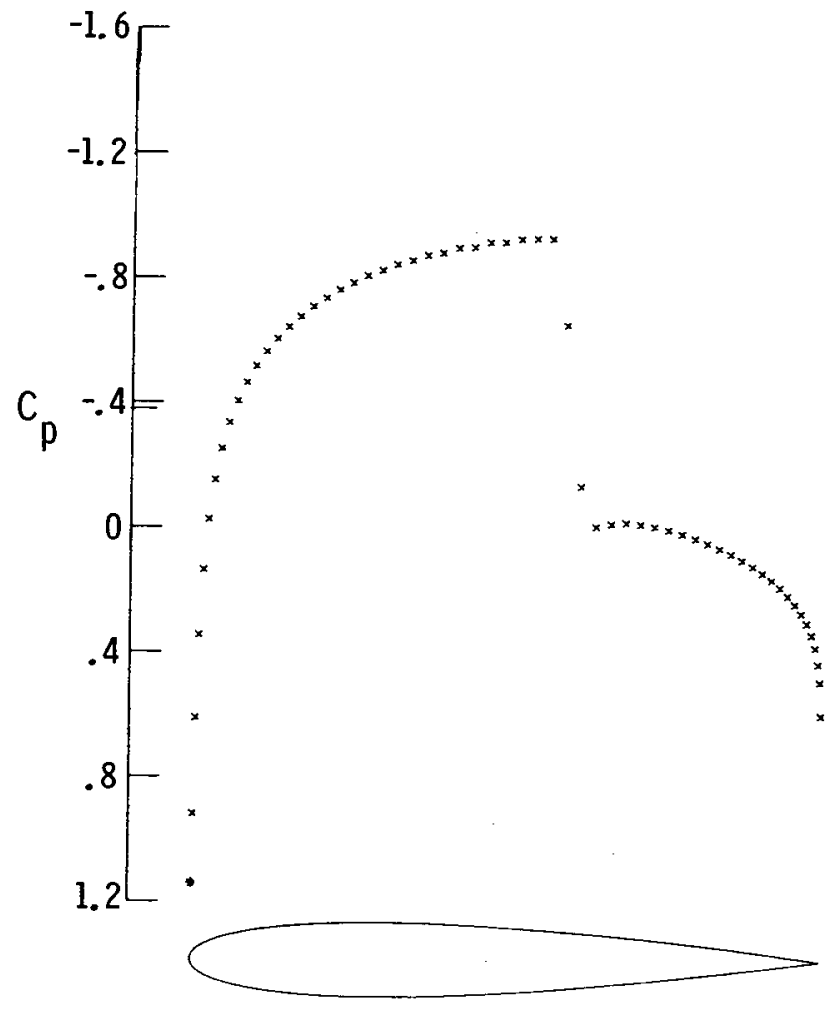

(b) Computed with FLO 52-S. $c_{l}=0.0000 ; c_{d}=0.0190$ $c_{m}=0.0000 ; 120 \times 34$ mesh; 2000 cycles; residual of
$0.165 \times 10^{-6}$.

Figure 11. Pressure coefficient for NACA 0012 airfoil at $M_{\infty}=0.82$ and $\alpha=0^{\circ}$. 


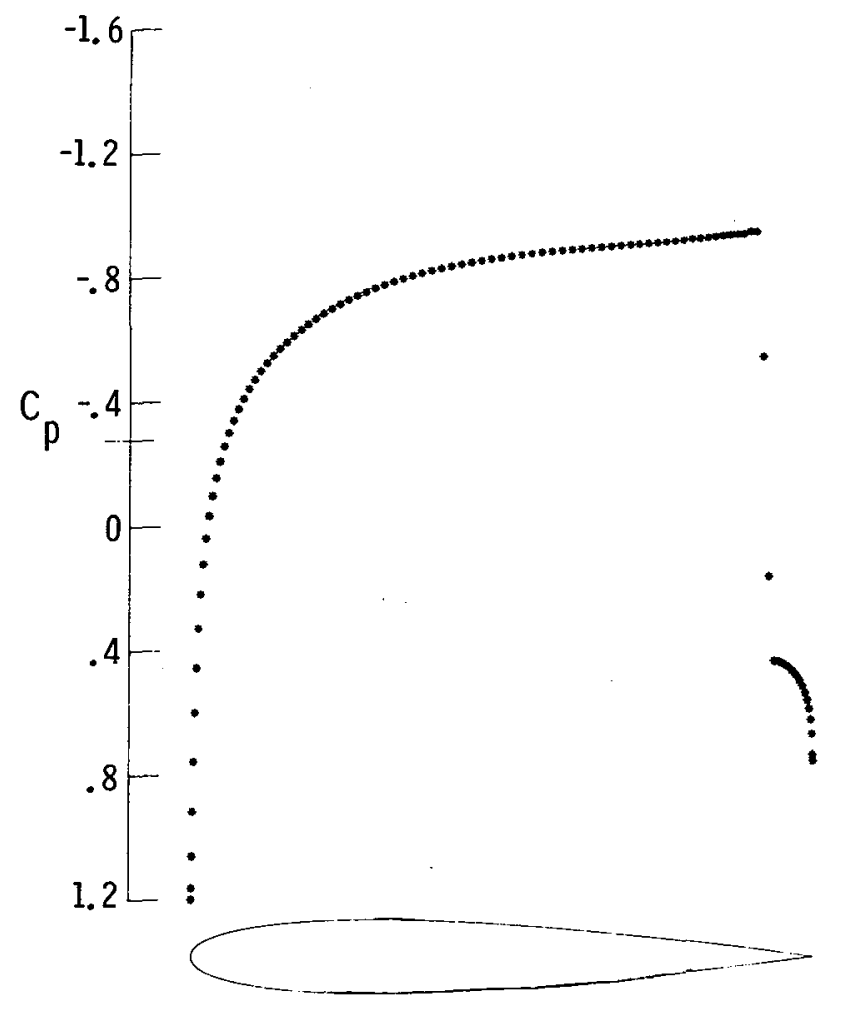

(a) Computed with FLO 36. $c_{l}=0.0000 ; c_{d}=0.0854$; $c_{m}=0.0000 ; 192 \times 32 \mathrm{mesh} ; 98$ cycles; residual of $0.822 \times 10^{-12}$

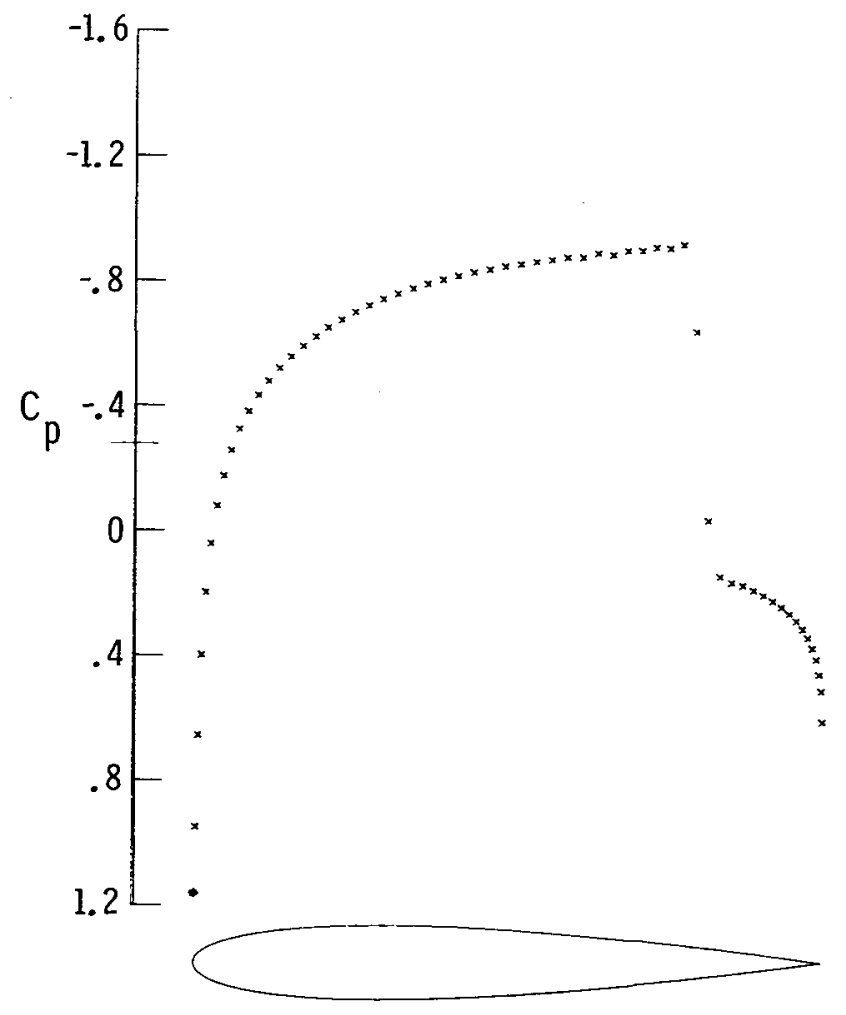

(b) Computed with FLO 52-S. $c_{l}=0.0000 ; c_{d}=0.0577$; $c_{m}=0.0000 ; 120 \times 34$ mesh; 2000 cycles; residual of $0.124 \times 10^{-6}$.

Figure 12. Pressure coefficient for NACA 0012 airfoil at $M_{\infty}=0.86$ and $\alpha=0^{\circ}$. 


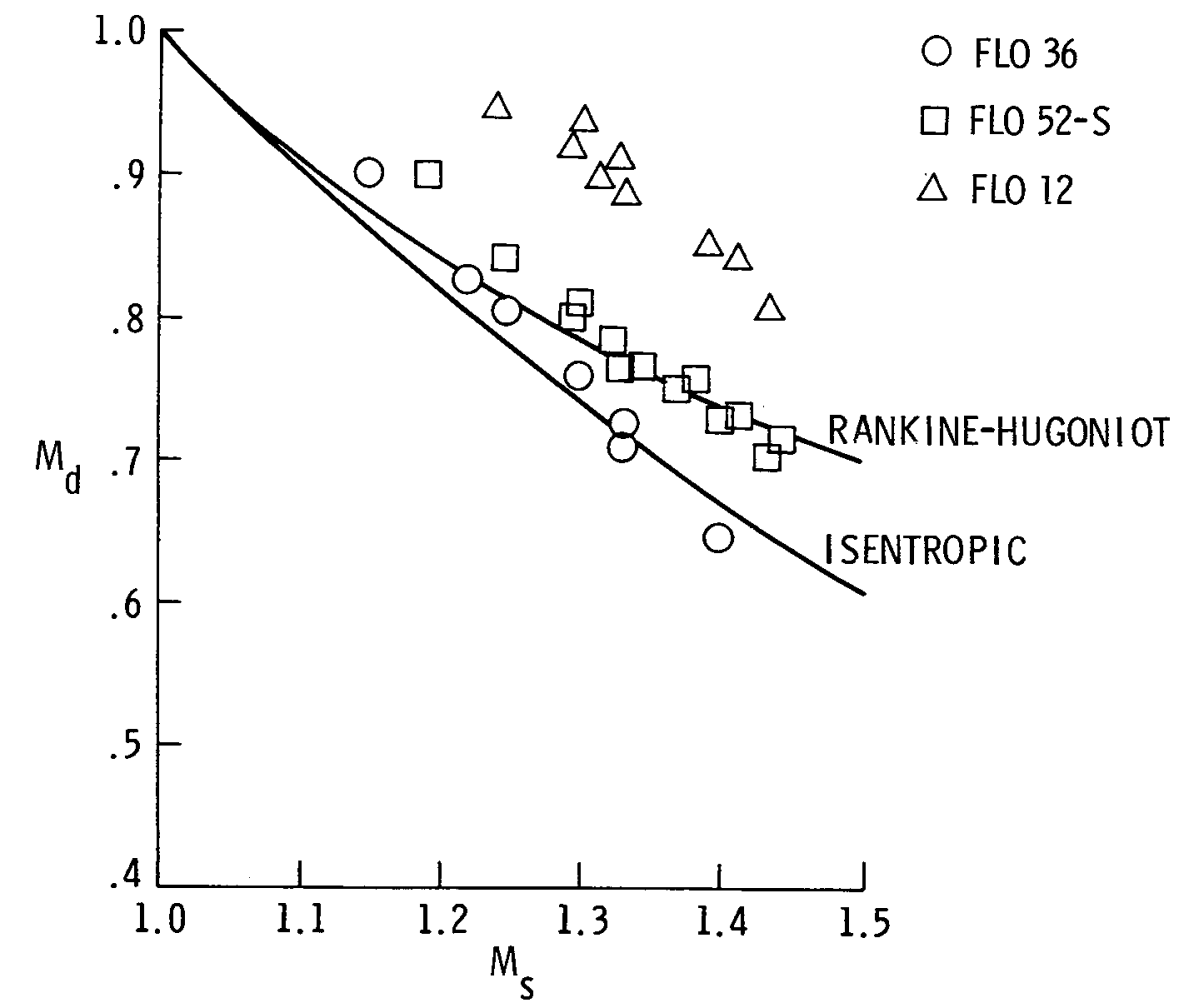

Figure 13. Shock jump conditions computed with FLO 36, FLO 52-S, and FLO 12 compared with exact jump.
O FLO 36

$\square$ FLO 52-S

$\triangle \mathrm{FLO} 12$

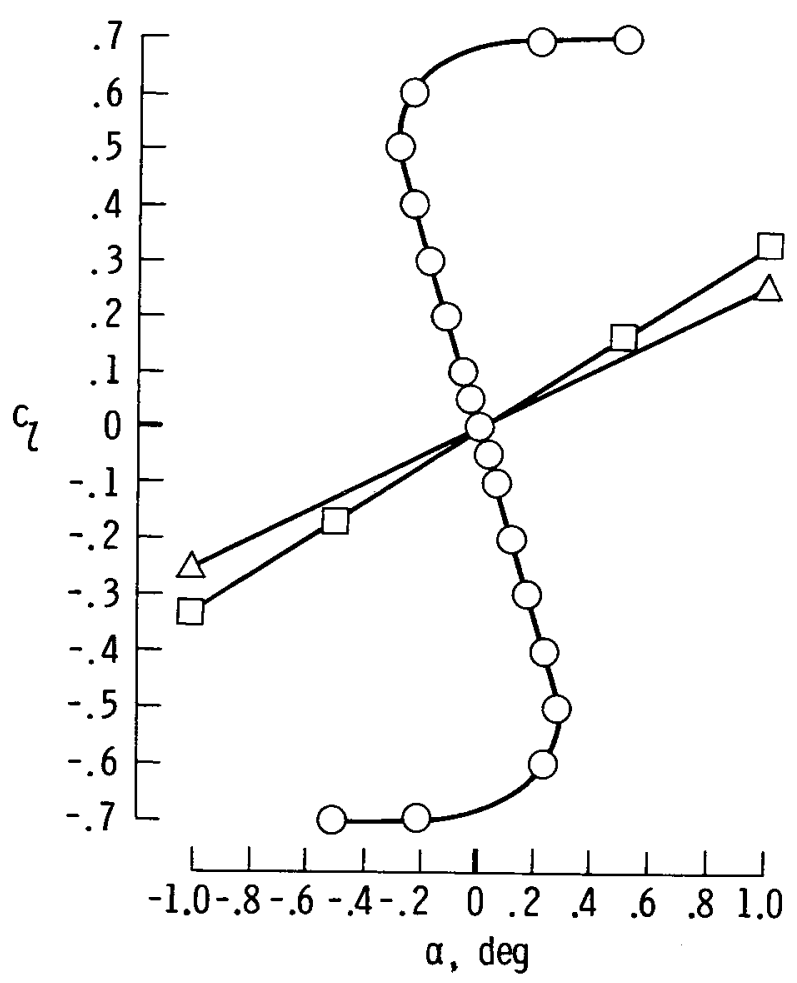

Figure 14. Lift curve obtained with FLO 36, FLO 52-S, and FLO 12 for NACA 0012 airfoil at $M_{\infty}=0.83$. 


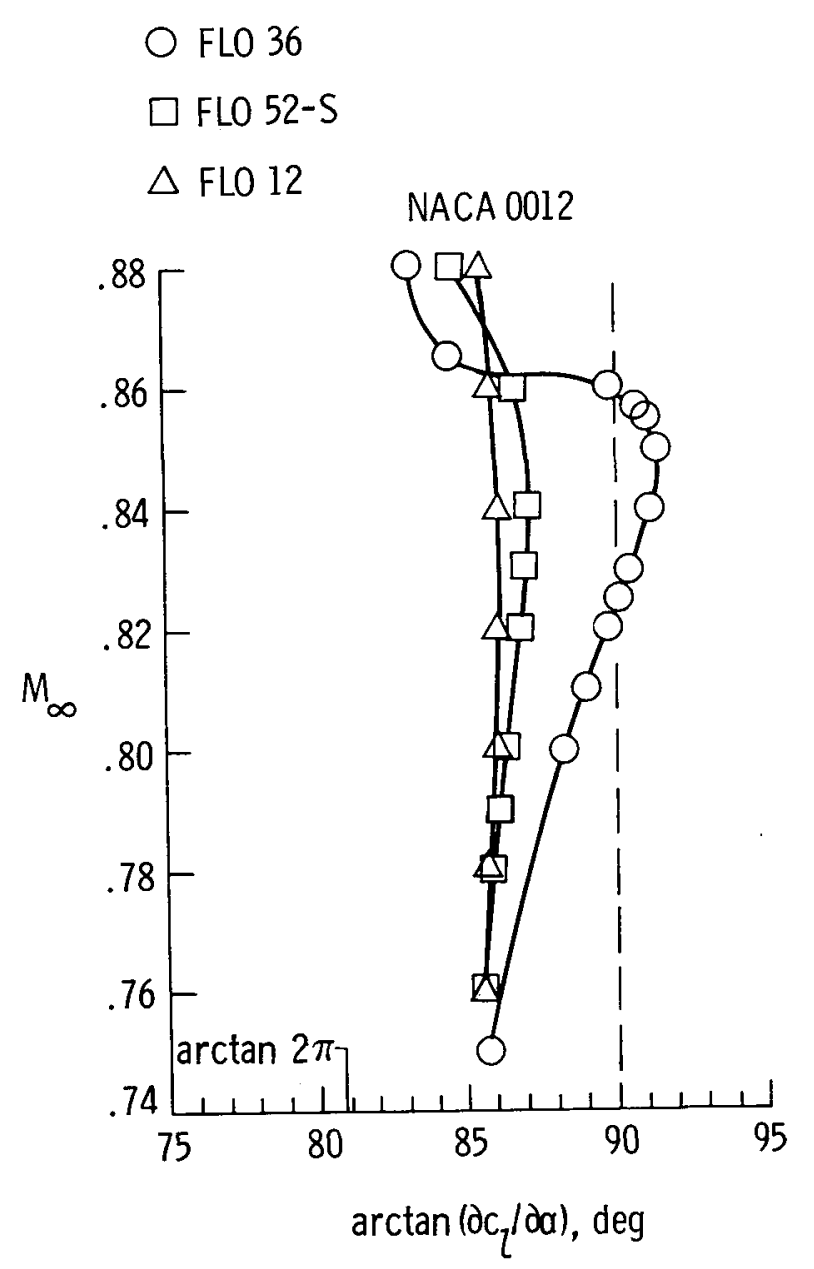

Figure 15. Angle made by lift curve with abscissa at zero lift and different Mach numbers for various formulations.

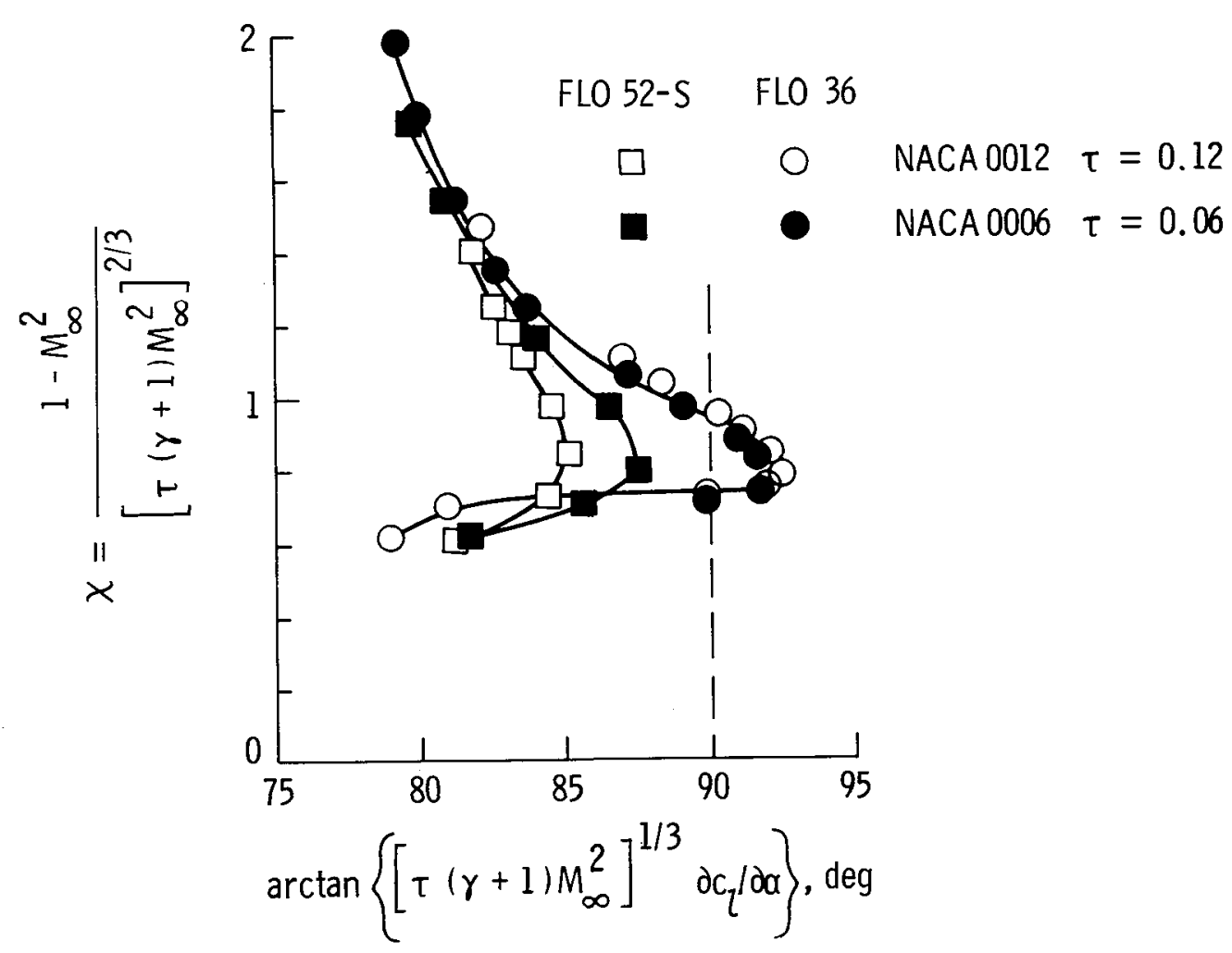

Figure 16. Angle made by lift curve in terms of transonic similarity parameters for two affine profiles for conservative potential and Euler formulations. 


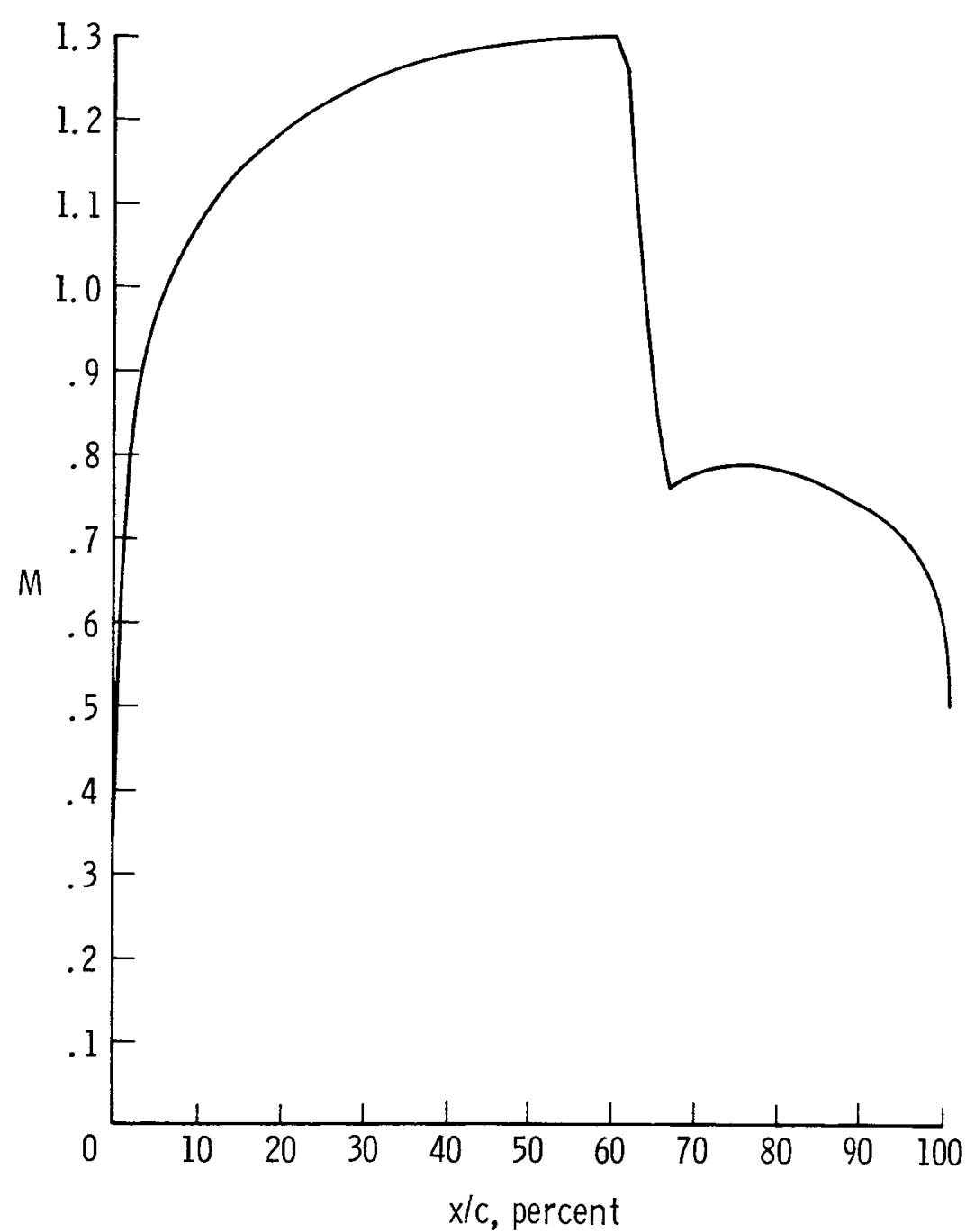

Figure 17. Computed surface Mach number for NACA 0012 airfoil at $M_{\infty}=0.820$ and $\alpha=0^{\circ}$ with FLO 36 .

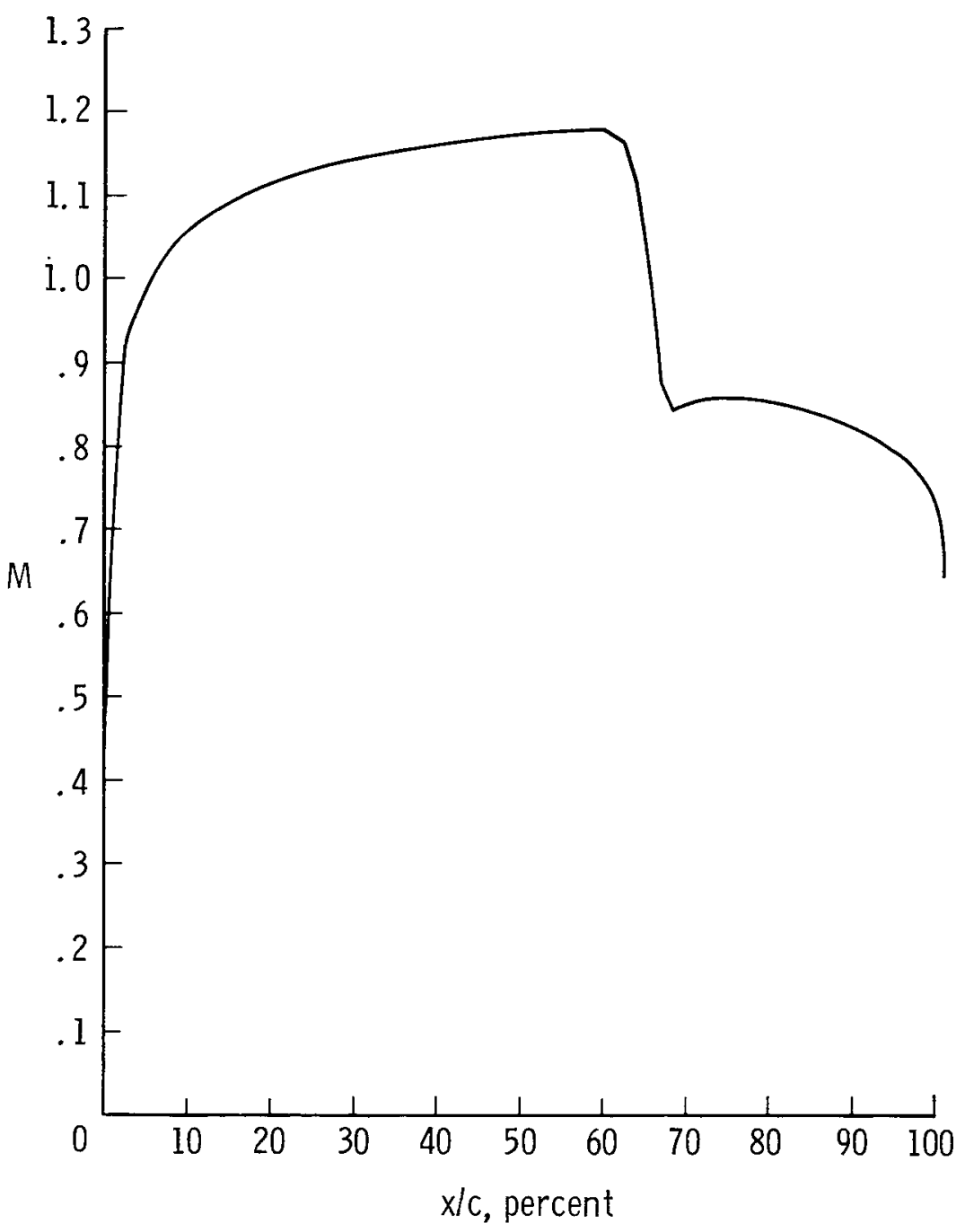

Figure 18. Computed surface Mach number for NACA 0006 airfoil at $M_{\infty}=0.879$ and $\alpha=0^{\circ}$, with FLO 36. 


National Aeronautics and Space Administration

Washington, D.C. 20546

Official Business

Penalty for Private Use, $\$ 300$
Postage and Fees Paid National Aeronautics and Space Administration NASA-451

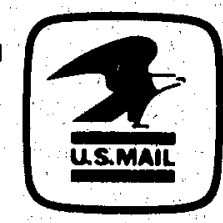

DO NOT REMOVE SLIP FROM MATERIAL

Delete your name from this slip when returning material to the library.

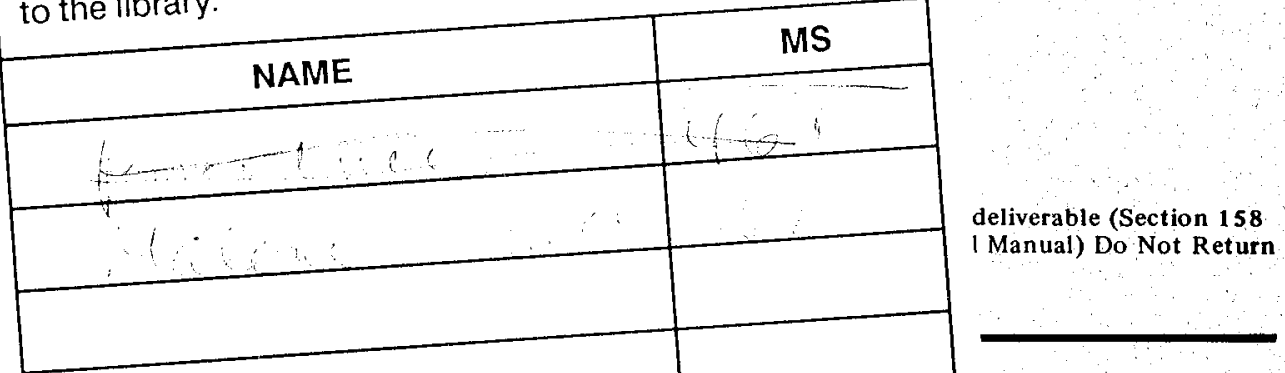

